\title{
SOLPS Modelling of ASDEX Upgrade H-mode Plasma
}

\author{
A.V.Chankin, D.P.Coster, R.Dux, Ch.Fuchs, G.Haas, A.Herrmann, L.D.Horton, \\ A.Kallenbach, M.Kaufmann, Ch.Konz, K.Lackner, C.Maggi, H.W.Müller, J.Neuhauser, \\ R.Pugno, M.Reich, W.Schneider
}

Max-Planck-Institut für Plasmaphysik, EURATOM Association, Garching, Germany

\begin{abstract}
A low density H-mode plasma has been selected for detailed inter-ELM modelling by the SOLPS code package, with the coupled treatment of its plasma (fluid code B2) and neutral (Monte-Carlo code Eirene) parts. Good quality measured midplane density and temperature profiles, covering the pedestal region and stretching far into the SOL, as well as several other parameters and profiles measured in the divertor, have enabled testing the consistency of code solutions with experiment. Once the upstream, midplane profiles, have been fitted, and the global parameters (e.g. input power into the computational grid, radiated power) matched, the code reproduced experimental profiles and control parameters in the divertor with the accuracy within a factor 2. Deviations of modelled parameters from the experiment were found around the strike point position where most of the power was deposited on the target. The deviations are consistent among themselves and all point to one common problem with the modelling: the predicted divertor electron temperature is too low, and the density too high, compared with the experiment. The largest inconsistency between the code and experiment was in the magnitude of the peak $\mathrm{H}_{\alpha}$ radiation in the outer divertor, which was larger by a factor of 2 in the code simulations. In addition, the code predicts a somewhat higher sub-divertor neutral flux, but lower carbon impurity content in the edge plasma than in the experiment, as well as lower CIII emission. The discrepancy between $\mathrm{H}_{\alpha}$ profiles can to a large degree be attributed to profile effects: the simulated $\mathrm{H}_{\alpha}$ emission profiles are narrower than in the experiment, reflecting the tendency for the neutral-plasma mix to congregate excessively around the strike point in the modelling. At the same time, the integrated $\mathrm{H}_{\alpha}$ emission matches very well with the experiment.
\end{abstract}

Extensive sensitivity studies of the influence of variations in input parameters and assumptions of the code on the modelled divertor conditions have been conducted. They have not resulted in an identification of any SOLPS input/control parameters capable of removing the main disagreement between the code output and experiment. A possibility for parallel transport effects related to low collisionality to increase the effective plasma temperature near the strike point position, or for increased perpendicular transport by neutrals (due to some missing reactions in Eirene) to widen the target profiles, will be explored in the future. 


\section{Introduction}

SOLPS is the code package consisting of the fluid plasma code, B2, and a kinetic MonteCarlo neutral solver, Eirene, for the plasma edge of tokamaks including outer core edge, scrape-off layer (SOL) and divertor regions [1-4]. It has been extensively used to model edge plasmas of ASDEX Upgrade (AUG) and some other existing machines (see e.g. [5]), as well as for predictions of (mainly divertor) conditions in ITER (see e.g. [6]). Predictions for future machines, such as ITER, require identification of key physical mechanisms determining the behaviour of the plasma-neutral-impurity $\operatorname{mix}$ in the tokamak edge. It also requires knowledge of some arbitrary parameters, such as perpendicular plasma transport coefficients, which are difficult to know a priori but whose influence on the code solution may be crucial. The way to achieve both objectives is to learn from modelling of experimentally welldocumented plasmas in different machines, by reconstructing experimental signals and establishing key parameters to be used as inputs to the codes.

On ASDEX Upgrade, a generally successful match between modelling results and experimental data has been achieved in a number of important regimes (modes) of the tokamak operation. In [7,8], modelling of both L- and H-modes in various divertor configurations is described. For the practically important (for ITER) H-mode operation, after adjusting perpendicular transport coefficients to fit experimental profiles and identifying chemical sputtering coefficients necessary to reach the measured radiated power, good match with several experimental signals (e.g. within a factor of 2, for spectroscopic signals) has been achieved [7,8]. Still, it was felt that, for the given diagnostic facilities of AUG, more could be done for direct quantitative comparison between modelling and experiment provided the right 'target plasma' could be established. AUG is equipped with a very good set of edge diagnostics, including pedestal and SOL plasma density and temperature profiles, as well as many profiles and parameters in the divertor. This allows one to dramatically reduce the number of free input parameters used in the edge code modelling and thus to verify code solutions via more precise quantitative consistency checks with experiment.

This paper describes steady-state SOLPS modelling applied to one of the best diagnosed AUG H-mode plasma with low plasma density. The choice of the plasma density was necessitated by the desire to reduce the frequency of the ELMs, so that experimental data for the inter-ELM quasi-steady-state conditions could be collected, and subsequently modelled. The evolution of the edge plasma between ELMs causes a certain scatter (in addition to that caused by the instrumental technique and turbulent fluctuations) of points on the profiles of the plasma density, temperature and spectroscopic signals. This scatter was not very large in the experiment (the scatter of $n_{e}, T_{e}, T_{i}$ signals can be directly assessed from the profiles presented below), and statistically averaged data could be used as representative for the whole inter-ELM period. The input power into the numerical grid was corrected for the rise in average energy content of the plasma during ELMs. No attempt was made, however, to model the same plasma with time-dependent calculations.

Low density H-mode plasmas push some of the assumptions of SOLPS to the limit. This code, as well as most of other codes used for modelling of edge tokamak plasmas (e.g. EDGE2D [9-11], UEDGE [12]) assumes collisional plasma transport along the field lines, while anomalous transport coefficients are usually assumed for perpendicular transport. As the input power into the discharge increases, and provided the plasma density is not high, the edge plasma becomes less collisional. The usual practice in today's edge codes is to treat 
non-collisional effects by setting up various flux limits [13-15], e.g. by limiting the maximum parallel electron/ion heat flux to an energy flux carried by one-sided electron/ion Maxwellian distribution function. Such an approach, of course, provides only a crude treatment of collisionless effects and does not guarantee correct solutions, which may require solving the Fokker-Planck equation in the SOL, implemented e.g. in Vlasov-like or PiC (particle-in-cell) codes, as discussed in [15]. To establish the degree of uncertainty low collisionality introduces into the SOLPS results, a dedicated sensitivity study of the variation of the flux limits, as well as some other input parameters for the modelling, has been performed. This is described in section 7 of the present paper. The rest of the paper is organised as follows. Section 2 describes the basic experimental parameters of the $\mathrm{H}$-mode shot selected for the modelling. Section 3 gives details of power and particle balance calculations for the modelled plasma. Section 4 lists some specific assumptions adopted in the SOLPS modelling described here. Section 5 gives details of the fitting of experimental profiles at the outer midplane by the code. In section 6 comparison between experimental and modelled parameters characterizing conditions in the divertor is presented. Section 8 contains some comments on the measured and calculated ion target fluxes and $\mathrm{H}_{\alpha}$ emission. Finally, section 9 gives a summary of the work.

\section{Basic experimental parameters of the selected H-mode shot}

The selected H-mode shot was a single-null discharge in deuterium, with the X-point at the bottom, where the ASDEX Upgrade Lyra IIb divertor was installed. The material of the divertor target plates was carbon (CFC), and the vessel is made of stainless steel (some invessels structures, including the upper divertor, have been covered by tungsten coated tiles, but the tungsten content in the plasma, as well as of iron, was found to be very small). Helium glow discharge cleaning is routinely used between shots, so some amount of helium is always present in the plasma. The magnetic configuration with the plasma current $0.8 \mathrm{MA}$ and toroidal field $2.0 \mathrm{~T}\left(q_{95} \approx 5\right)$ is presented in Fig. 1a, for the time slice of $4.13 \mathrm{~s}$.

Time traces of the main experimental signals are shown in Fig. 2. The time slice selected for the modelling was towards the end of the first, lower density H-mode phase with type I ELMs of $\sim 50-60 \mathrm{~Hz}$ frequency. The line-averaged density was $\approx 5.8 \times 10^{19} \mathrm{~m}^{-3}$, corresponding to $\approx 60 \%$ of the Greenwald density limit. The density was maintained by the Neutral Beam Injection (NBI) of 5.1 MW, without gas fuelling. The cryo-pump was switched on (see Sec. 3 on the detailed particle balance).

\section{Power balance, particle balance}

One of the most important parameters for the SOLPS solution is the total input power into the computational mesh (or 'grid') shown in Fig. 1b. This power was calculated by subtracting various power fluxes that occur outside the volume of the grid, from the total input power of 5.1 MW into the discharge. Corrections have been made for the beam shine through (beam particles not absorbed by the plasma), $0.2 \mathrm{MW}$, fast ion losses from the plasma, $0.4 \mathrm{MW}$, increase in the plasma energy content in-between ELMs $(d W / d t), 0.7 \mathrm{MW}$, and radiation power in the inner core, inside of the mesh, 1.1 MW. The power input into the computations mesh was thus reduced to $2.7 \mathrm{MW}$. 
The experimental radiated power inside the grid region is another important parameter in the SOLPS modelling, that must be closely matched. It was calculated using data from a number of bolometers viewing the plasma. After subtracting radiated power inside of the separatrix, 1.1 MW, from the total radiated power of $2.4 \mathrm{MW}$, radiated power of about 1.3 MW inside the grid is obtained. (Radiated power in the outer core region between the inner core boundary of the grid and separatrix position can be neglected as small). Correction for the ELM contribution, established by using fast bolometer data, reduces this radiated power to 1.15 MW, for inter-ELM periods. Both in the experiment and the SOLPS modelling, the bulk of the radiated power inside of the grid came from the divertor, being split more or less equally between impurity and deuterium radiation.

The detailed particle balance can be established by the use of ionization pressure gauges measuring total neutral gas flux densities at various positions outside of the plasma, as shown in Fig. 3a. The total pump-out of neutrals from the system can be found from the neutral flux in the pumping region $\left(4.5 \times 10^{21} \mathrm{~m}^{-2} \mathrm{~s}^{-1}\right.$, in terms of deuterium atom flux, hence, each molecule is counted as two atoms; molecules dominate the atom-molecular mix in areas shielded from the direct influence of atoms coming from the plasma). Pumping is dominated by the cryopump, operating at $\approx 100 \mathrm{~m}^{3} \mathrm{~s}^{-1}$ pumping rate (the turbo-molecular pump system contributes only $\approx 10 \mathrm{~m}^{3} \mathrm{~s}^{-1}$ ).

In the majority of SOLPS runs described in this paper, a simplified particle balance was used, by setting an absorption coefficient of the 'pump' in Eirene (see Fig. 3b) so as to remove the neutral source in the plasma originating from the NBI (equivalent to $\approx 80 \mathrm{~A}$, or $5 \times 10^{20}$ atoms per second). This determined the total particle throughput (combined ions + neutrals flow across the magnetic surfaces) and the value and spatial profile of the plasma diffusion coefficient $\mathrm{D}_{\perp}$, necessary to match experimental density profiles. In this simplified particle balance actual experimental pumping of neutrals by the pumps was ignored, and only the consistency between experimental sub-divertor neutral flux and the modelled one, invoked from calculating particles crossing the 'measurement surface' placed underneath the horizontal target plate in Eirene, was monitored. The experimental sub-divertor neutral flux is one of the main parameters characterizing, and closely related to, the conditions in the divertor. Also ignored was the large drop of neutral flux from the sub-divertor region to the pumping region, which in more refined neutral description necessitated the introduction of an extra 'baffle' in Eirene (see Fig. 3b), roughly accounting for blockages for the neutral exchange between sub-divertor and pumping regions by various material divertor and wall structures.

Such a simplified particle balance as described above would be applicable if neutral recycling from the walls was $100 \%$, on average. In reality, the removal rate of neutrals by the pumping system was found to be considerably higher than the particle input from the NBI source. The neutral flux of $4.5 \times 10^{21} \mathrm{~m}^{-2} \mathrm{~s}^{-1}$ (by molecules at room temperature) combined with the pumping rate of $100 \mathrm{~m}^{3} \mathrm{~s}^{-1}$ leads to a neutrals removal rate of $\approx 275 \mathrm{~A}$, considerably exceeding the particle input into the system from the NBI. One therefore has to conclude that in the series of low density H-mode discharges, part of which was the modelled shot \#17151, a substantial outgassing of the walls took place, with the released working gas (deuterium) subsequently being removed by the pumping system. Hence, a significant internal circulation of neutrals between the walls and the plasma must be taken into account in the detailed neutral model. In the present work, wall outgassing was modelled by a poloidally uniform gas puff. Further details are described in section 7. It is important to stress, however, that SOLPS runs resulted in almost the same divertor conditions, regardless of whether the simplified or 
more realistic neutral description was used in Eirene. The most important parameters for the solution were found to be the total input power into the grid, separatrix density and the radiated power. These parameters determine to a large degree the recycling flux in the divertor which exceeds the pumped flux by two orders of magnitude. At the same time, outgassing from the wall and neutral transport through the gaps in the target plates to the subdivertor region, then further to the pumping region and their subsequent removal by the pumping system, were of secondary importance for divertor parameters.

\section{Specific assumptions of the code modelling}

SOLPS calculations described in this paper have been performed on a $48 \times 18$ computational mesh presented in Fig 1b. Test cases on a finer, 96x36 mesh, have also been run, but the results were found to be virtually identical to those with the coarser mesh. The simulated plasma consisted mainly of deuterium and carbon, with a possibility to add helium $(\mathrm{D}+\mathrm{C}+\mathrm{He}$ cases). In the experiment, small fractions of hydrogen and a somewhat larger fraction of helium were present in the plasma. It is in principal possible to determine the plasma mix in AUG, but this has not been done for the analyzed shot \#17151. The effect of adding helium in the simulations is described in Sec. 7.

In the modelling, carbon was produced self-consistently from physical and chemical sputtering, had zero recycling at the targets, private flux and SOL boundaries. The physical sputtering rates for both ions (including their acceleration in the Debye sheath) and neutrals were taken from the TRIM database [16,17], however, physical sputtering was relatively unimportant compared to the chemical sputtering due to low plasma temperatures. Constant chemical sputtering rates, typically in the range 1-3\%, have been used for carbon production. As in many other machines, due to continuous sputtering and redeposition, carbon layers, rich with trapped deuterium, were found on all the surfaces exposed to the plasma. In the modelling, therefore, carbon was chosen to be the material of all structures interacting with the plasma. The chemical sputtering rate was a free parameter in the calculations, adjusted to match the experimental total radiated power inside of the computational mesh.

Neutrals were treated self-consistently by Eirene, both inside (using known interaction reactions with ions and electrons) of the grid and outside of it, near the walls and in the subdivertor and pumping regions. Charged particles, on the other hand, were naturally present only inside of the grid. Once they reached private flux and SOL boundaries, a 'leakage' from the grid, at the rate of $1 \%$ of the parallel flux $n c_{s}$ ( $c_{s}$ is the ion sound speed), was applied. The particle content within the grid was fully or partly (depending on the chosen recycling coefficient) compensated by a return flow of working gas consisting of a mixture of atoms with local ion temperature and molecules at wall temperature. The exact percentage of the 'leaked' particles was found to be not an important parameter in the SOLPS modelling, for as long as this percentage wasn't high. Its variation resulted in some variation of density decay length near the grid boundaries, while the charged particle flux was little affected. 100\% recycling was assumed at the outer SOL boundary, private region and also at the target plates. Normal sheath boundary conditions were used at the (vertical) targets interacting with the plasma. A case with the uniform gas puffing from the wall, intended to simulate wall outgassing during the discharge, is described in Sec. 7.

The list of reactions included in Eirene in the SOLPS runs described in this paper is the following: ionization of deuterium atoms and molecules, carbon and helium atoms; charge- 
exchange between deuterium, carbon and helium atoms, on the one hand, and deuterium ions, on the other; dissociation of molecules; elastic collisions between molecules and helium atoms, on the one hand, and deuterium ions, on the other; volume recombination, including three-body recombination.

At the core boundary of the grid, the total ion + neutral flux was monitored and, in the converged solutions, was equal to the total particle input from the NBI $(\approx 80 \mathrm{~A}$, in equivalent units). In all calculations described here, the two main parameters most affecting the solution were total input power crossing the core boundary and separatrix electron density. The specified separatrix density was maintained by a feedback loop which varied the core boundary density in response to deviations in the separatrix density. The control over the separatrix density was thus achieved via variations in the density gradient between the inner core and separatrix positions and the particle flux through the separatrix. Transport coefficients were kept constant in time, but could be varied radially and, to a certain extent, poloidally (see below).

In most of the cases described here, input power into the grid was equally shared between ions and electrons. In Sec. 7 the impact of the variation in the power sharing on the solution is discussed.

There is a wide variety of opinions on the possible values of flux limits for parallel ion and electron heat fluxes [13-15]. They are used in the codes for smooth transition from a collisional regime where the parallel heat fluxes are given by Spitzer-Härm to a kinetically limited term as the collisionality drops. In the present modelling, the electron heat flux limit was chosen to be 0.3 (heat flux limited to $0.3 n_{e} T_{e} v_{t h, e}$ ), and the ion flux limit - to 1.0 (heat flux limited to $\left.n_{i} T_{i} v_{t h, i}\right)$. These flux limits have been, however, varied in a separate series of runs, and the results are presented in Sec. 7 .

In the modelling, all transport coefficients were assumed to be inversely proportional to the local toroidal field, $\sim 1 / \mathrm{B}$, within each magnetic surface. In addition to this, they could be individually specified on each surface (i.e. radially varied). Natural ballooning of the plasma transport due to field line compression on the outer, low field side of the plasma has occurred with the fluxes, e.g. particle flux $-D_{\perp} \nabla n$, being larger on the low field side (larger $-\nabla n$ ), for the same $D_{\perp}$. This ballooning was further amplified by the $\sim 1 /$ B dependence of the transport coefficients. Sensitivity of the code solutions to variations in the degree of ballooning of transport coefficients is discussed in Sec. 7.

Perpendicular (radial) viscosity was introduced in all SOLPS cases via the anomalous viscosity coefficient $\eta_{\perp}=0.5 n_{i} m_{i} D_{\perp}$, where $n_{i}$ and $m_{i}$ are density and mass of each ion species present in the plasma. Its influence on SOLPS solutions was not significant, as was established by varying the numerical coefficient on the right hand side of the above formula from 0 (no perpendicular viscosity) to 2 . This may be related to relatively small ion parallel velocity in the SOLPS solutions for the main SOL region, with typical Mach numbers of the parallel flow $\sim 0.1$.

Finally, the SOLPS version 5.0 was used in the calculations presented here, with B2.5 fluid plasma code and the Eirene version dated 1996. Most of the cases were run without drifts 
since their inclusion introduced severe numerical problems, leading to a dramatic reduction in the required time step.

\section{Fitting experimental pedestal and SOL profiles}

Good quality high resolution edge profiles of electron density and temperature have been obtained for $\# 17151$ by a number of diagnostics, covering the H-mode pedestal region and extending into the SOL. In addition, ion temperature was measured by a new edge chargeexchange system using a Lithium beam. Experimental details of these measurements can be found in [18].

Figure 4, replicated from [18], shows experimental $n_{e}, T_{e}$ and $T_{i}$ profiles together with the fits to the data using SOLPS modelling without drifts. The profiles have been collected in the time window $3.5-4.2 \mathrm{~s}$. For $n_{e}$ and $T_{e}$ profiles, an ELM synchronization was applied, measuring only data points taken during the time interval from 5 to $0.5 \mathrm{~ms}$ before each ELM. For $T_{i}$, data points have been collected randomly with respect to ELMs onset, but the data during ELMs themselves have been excluded. All positions in Fig. 4 are mapped to the outer midplane.

Also shown in Fig. 4 are radial profiles of perpendicular anomalous diffusion coefficient $\mathrm{D}_{\perp}$ and electron and ion heat conductivities $\chi_{e, i}$ that were used in SOLPS to reproduce the experimental profiles. Since ballooning of transport coefficients, $\sim 1 / B$, was used in the modelling, the data presented give averages over flux surfaces. Profiles of the transport coefficients clearly show the existence of a transport barrier inside of the separatrix. The profile of the ion neoclassical heat conductivity is also shown in Fig. 4 for comparison. In the transport barrier region, $\chi_{e}$ exceeds $\chi_{i}$ by a factor $\sim 4$, which, however, doesn't translate into the same inequality of radial power fluxes owing to the presence of convective power fluxes $5 / 2 \Gamma T_{e, i}$ (and note that $T_{i}>T_{e}$ ). There is nevertheless a certain redistribution of total power fluxes in favour of the electron channel due to ion-electron equipartition, from the initial equal sharing of the powers at the inner core boundary in most of the code runs described in this paper. The effect of the variation of the power sharing on divertor conditions is discussed in Sec. 7.

It is to be noted that the interpretive SOLPS modelling, with respect to the fitting of experimental outer midplane $n_{e}, T_{e}$ and $T_{i}$ profiles, leaves a large degree of freedom regarding a possible choice of theoretical models for the perpendicular transport. Instead of using $\mathrm{D}_{\perp}$ for the particle transport one could equally use e.g. a combination of $\mathrm{D}_{\perp}$ and a pinch velocity. Moreover, there is a growing evidence to suggest that turbulent transport in the SOL may be non-local and caused by radial advection of blob-like filamentary structures (see. e.g. [19]). This however is outside of the scope of the present study, where the chosen transport coefficients were only used to repeat as well as possible experimental radial upstream profiles, in order to create boundary (upstream) conditions for simulating the plasma in the divertor. The predictive power of SOLPS was therefore almost exclusively in its simulation of divertor conditions.

The separatrix density at the outer midplane, which was an input parameter in these SOLPS calculations, was set at $1.6 \times 10^{19} \mathrm{~m}^{-3}$. This choice was dictated by considerations of the best match to both $T_{e}$ and $n_{e}$ experimental profiles. In the experiment, owing mainly to an 
insufficient accuracy in determining the separatrix position (a few $\mathrm{mm}$ precision is required), the exact position of $T_{e}$ and $n_{e}$ data points cannot be established. In the code calculations, however, given the constraints imposed by the known power through the core boundary of the grid, and, to a lesser extent, by the known radiated power, the wrong choice of the separatrix $n_{e}$ would lead to a mismatch between measured and calculated $T_{e}$ profiles. This will be illustrated in Sec. 7 of the paper.

Electron and ion collisionality parameters, defined according to [13], Eq. (4.105), as $v_{e e}^{*} \equiv L / \lambda_{e e} \approx 10^{-16} n_{e} L / T_{e}^{2}$ and $v_{i i}^{*} \equiv L / \lambda_{i i} \approx 10^{-16} n_{e} L / T_{e}^{2}$, are plotted in Figs. 5a,b for the case shown in Fig. 4 . As a characteristic parallel length $L$, the product $\pi q_{95} R$, with $q_{95}=5$ being safety factor at the surface containing $95 \%$ of the poloidal magnetic flux and $R=1.7 \mathrm{~m}$ - major radius of the plasma, was used for all profiles. Figure 5a shows the collisionality parameters along radial distance at the outer midplane position, and along the outer divertor target. All positions are mapped to the outer midplane. Figure $5 \mathrm{~b}$ shows the collisionality parameters plotted against parallel distance from the inner target (negative distances) to the outer (positive distances) along the first SOL flux tube. One can see that the electron collisionality parameter is substantially above 1 across the whole SOL, including at the separatrix position near the outer midplane. Electrons therefore can be considered collisional. At the same time, the ions are weakly collisional upstream, being only marginally collisional near the separatrix. Close to the divertor, ions, as well as electrons, become collisional. For parallel heat conduction, however, due to the importance of contributions from highly energetic tails of the Maxwellian distribution (typically with energies of $\sim 3-5 T_{e, i}$ ), both ions and electrons can be considered as collisionless in the main SOL region, and the flux limits discussed in the previous section must be applied. Closer to the divertor, the plasma becomes much more collisional owing to a simultaneous rise in density and a drop in temperatures.

Overall, it is difficult to predict the full extent of the contribution of kinetic effects to the results of the SOLPS modelling in such a complex situation. Their impact on the divertor solutions is assessed in Sec. 7 by varying heat flux limits for both ions and electrons.

\section{Matching divertor conditions: identifying an inconsistency}

In SOLPS calculations, the input power into the grid was originally $2.7 \mathrm{MW}$, in agreement with experiment. The experimental radiated power inside of the grid, $1.15 \mathrm{MW}$, was matched by assuming chemical sputtering rate of $1.6 \%$. One of the most important parameters to be matched by the modelling is the power deposition at the outer divertor target. Figure 6 shows experimentally determined power deposition profiles at the outer target together with those calculated from 3 different SOLPS cases.

Power deposition from the IR camera represents the signal averaged over 50 measured profiles in-between two representative ELMs separated by about $28 \mathrm{~ms}$. The background signal due to radiation has been subtracted. The Langmuir probe profile has been obtained during vertical plasma sweeps over stationary triple probes imbedded in the target. The probes measured ion saturation current density, $j_{s a t}$, and electron temperature. By using standard procedure in evaluating electron density $n_{e}$ from this data, the power flux density was then calculated as $\gamma c_{s} n_{e} T_{e}$, with $c_{s}$ being the ion sound speed and $\gamma=8$. 
One of the reasons for the disagreement between the IR and Langmuir probes data, which will be discussed later in this section, could be low biasing voltage of the probes, which was set at $-36 \mathrm{~V}$. The SOLPS calculated target power, for the case of $2.7 \mathrm{MW}$ of input power, is substantially below that measured by the IR camera. Part of this difference can perhaps be explained by the fast ion losses which were not included in SOLPS. The other part must have come from the unrealistic power sharing between the outer and inner targets calculated by the code where classical drifts $(\mathrm{E} \times \mathrm{B}, \nabla \mathrm{B}$ and centrifugal drifts) were not included. In the code, the ratio of powers directed into outer and inner divertors was 1.57:1, substantially below typical experimental values (see just below), hence, the calculated outer target power was underestimated. The outer target power could be increased by running SOLPS with drifts included into the B2 plasma code (referred to then as B2.5), as shown in Fig. 6. The ratio of the powers is then increased to $2.87: 1$. This is the expected result of adding drift terms into the edge modelling (see e.g. review paper [20] and recent experimental results [21] as well as EDGE2D modelling in JET $[22,23])$. Cases with drifts, however, have been affected by numerical instabilities severely limiting the maximum time step in the calculations leading to a large increase in the total run time. Moreover, for this relatively high input power low density plasmas, drifts could only be switched on in the SOL, divertor and extreme outer core regions (but switched off in the bulk of the core region), again for the sake of numerical stability. In the remainder of this section, simulated results for the case with drifts will be shown. In the next section, where the emphasis will be mostly on the conditions in the outer divertor, a non-drift case with slightly higher input power, 3.0 MW, will be used as a reference case in the sensitivity study. The power deposition profile at the outer target for this case is also presented in Fig. 6.

All profiles in Fig. 6 are plotted against the coordinate 's' directed along the targets. The SOLPS calculated powers are given in the first place versus distances from the separatrix and later re-calculated onto the target ' $\mathrm{s}$ ' coordinate using the magnetic equilibrium reconstruction. The later however is not very precise, resulting in spatial shifts between experimental and calculated profiles in some cases. All calculated profiles therefore have been shifted by $1.4 \mathrm{~cm}$ outward to ease their comparison with experimental profiles.

Power deposition profiles at the inner target are shown in Figure 7. From SOLPS calculations, only the results of the case with drifts are presented. The power deposition profile deduced from the IR camera, shown in Fig. 7, apparently suffers from the presence of re-deposited carbon layers at the inner target, resulting in the peak power deposition at the inner target substantially, by a factor of 2 , exceeding that at the outer target (when assuming pure carbon surface), followed by periods of negative power. IR-invoked power profiles are therefore much less reliable at the inner target, and require incorporation of a proper surface model correctly reflecting the width, thermal properties and spatial distribution of redeposited layers. As for the outer target, experimental power profiles between ELMs did not exhibit large variations, provided the data is not taken just after an ELM. Hence, these profiles can be considered reliable.

In-between ELMs, the plasma is strongly detached at the inner target, both in experiment (as can be seen from CIII and $\mathrm{H}_{\alpha}$ emission profiles not shown here) and calculations. In the modelling, $T_{e}$ drops to $0.4 \mathrm{eV}$ at the strike point position, but then rises to $T_{e, \max }=2.2 \mathrm{eV}$ at the position of maximum $n_{e}$, which must however be too high for the complete detachment which is usually associated with temperatures $T_{e}<1 \mathrm{eV}$, when recombination becomes most effective. 
Other parameters at the outer target, both measured and calculated using the case with drifts, are shown in Figure 8. The $T_{e}$ profiles, from SOLPS and Langmuir probes, are not too different from each other, but the calculated $n_{e}$ values are much higher than in the experiment (in addition, a feature in the calculated profile with $n_{e}$ rising in the private regions can be identified, which must be attributable to drifts). Due to the low biasing voltage, mentioned earlier, Langmuir probe $n_{e}$ could be underestimated, provided $j_{s a t}$ was not reached. On the other hand, the probes' measured $T_{e}$, which is below $18 \mathrm{eV}$ across the whole target profile, is substantially below the absolute value of the biasing voltage of $36 \mathrm{~V}$ multiplied by electron charge $e$. Hence, if one was to trust the measured target $T_{e}$, at least a quasi-saturation had to be reached by the probes, leaving little room for a possible underestimate of the probe $n_{e}$. Since a large discrepancy in $n_{e}$ is nevertheless present, one should suspect the probes $T_{e}$ of being underestimated. This suspicion is supported by the very low target power deposition calculated by the probes as shown in Fig. 6 .

The reasons for the probes not measuring $T_{e}$ correctly could be distortions of the electron Maxwellian distribution introduced by energetic electrons coming from the main SOL region where $T_{e}$ exceeds $100 \mathrm{eV}$ near the separatrix, or even a bi-Maxwellian electron distribution, with one part of the distribution being at a temperature substantially exceeding that evaluated by the probes. Given the relatively modest target $T_{i}$ values predicted by the code near the separatrix, an alternative explanation via insufficiently high factor $\gamma$ in the formula $\gamma c_{s} n_{e} T_{e}$ evaluating the power flux at the target, due to $T_{i} \gg T_{e}$, seems unlikely: $\gamma=8$ assumes $T_{i}=T_{e}$. Further experiments are planned in AUG, with the repetition of the same shot, but with higher biasing voltage for Langmuir probes and recording single probe $\mathrm{I}-\mathrm{V}$ characteristics to more precisely determine electron temperature. If, however, the peak target $T_{e}$ in the experiment is really much greater that the measured one, then secondary electron emission can become an issue.

The above discrepancies between measured and calculated $T_{e}$ and $n_{e}$ profiles, as well as between power deposition profiles invoked from the IR camera and Langmuir probes, can be explained by assuming that both probes $T_{e}$ as well as temperatures calculated by SOLPS are significantly underestimated. With respect to the SOLPS results, this implies that the averaged electron energy in experiment is significantly above $3 / 2 T_{e}$, where $T_{e}$ is taken from the code. This seems to be the only plausible explanation for the discrepancies, especially in light of other indirect evidence for a much hotter divertor, presented below.

One therefore has to conclude that the maximum SOLPS $n_{e}$ in the outer divertor must be much higher than in the experiment. For a given power deposition at the outer target, the match of the experimental power by the code is mainly achieved by raising density above the experimental value, whereas the temperature(s) are lower than in experiment. This is confirmed by the comparison between measured and simulated spectroscopic $\mathrm{H}_{\alpha}$ and CIII (at $465 \mathrm{~nm}$ ) line integrated emission profiles in the divertor, shown in Fig. 8 (lines of sight of this diagnostics are shown in Fig.9). The simulated CIII emission profile is much below the experimental one, whereas the peak in the simulated $\mathrm{H}_{\alpha}$ emission profile is larger by about a factor 2 compared with the experiment. The simulated $\mathrm{H}_{\alpha}$ profile is also much narrower than in experiment, while the integral $\mathrm{H}_{\alpha}$ emissions are almost the same in the modelling and experiment. Higher simulated $\mathrm{H}_{\alpha}$ emission than measured in the experiment implies a larger ion flux to the target caused by higher $n_{e}$ and lower $T_{e}$, for the same plasma pressure which is essentially determined by the pressure upstream, at the outer midplane. 
An attempt was made to raise the CIII emission in the modelling, so as to bring it closer to experimental values, by increasing the chemical sputtering coefficient by $64 \%$. This increased the total radiated power within the computational grid by $20 \%$, which was considered acceptable since this quantity is not very well defined in the experiment. The peak in the simulated CIII emission was, however, only increased by $18 \%$, far insufficient to match the experimental values.

The tendency for SOLPS solutions to predict denser and cooler divertor compared with experiment is further corroborated by comparing calculated and measured neutral fluxes. The calculated neutral flux obtained by counting all particles crossing the 'measurement surface' just below the target in Eirene in the sub-divertor volume, shown in Fig. 3b, was $7.3 \times 10^{22} \mathrm{~m}^{-2} \mathrm{~s}^{-1}\left(6.5 \times 10^{22} \mathrm{~m}^{-2} \mathrm{~s}^{-1}\right.$ for a corresponding non-drift case $)$, substantially above the experimental value of $3.5 \times 10^{22} \mathrm{~m}^{-2} \mathrm{~s}^{-1}$ (see Fig. 3a). The calculated neutral flux at the outer midplane $\left(8.1 \times 10^{20} \mathrm{~m}^{-2} \mathrm{~s}^{-1}\right)$ also exceeds the measured one. The discrepancy between subdivertor, as well as midplane, neutral fluxes can be reduced by using a more adequate neutral description in Eirene. This is described in Sec. 7. The discrepancy however is not completely eliminated, and the code still predicts a somewhat higher neutral flux in the sub-divertor region than in the experiment.

Finally, a mismatch between calculated and measured (obtained from the CHEAP code) carbon concentrations in the plasma also supports the view that SOLPS predicts too dense and cool plasma in the divertor. Experimental values of $\sim 1 \%$ in the core contrast with a much lower, $0.1-0.4 \%$, depending on the case, calculated carbon concentrations in the SOL and outer core regions. Since carbon radiation is more efficient at low $T_{e}$ (and high $n_{e}$ ), lower carbon concentrations are required in the code to radiate the same power as in the experiment. The bulk of the radiated power in the code, as well as in the experiment, comes from the divertor.

\section{Sensitivity studies of divertor conditions in the code: attempts to resolve discrepancies between experiment and modelling}

Evidence presented in the previous section suggests that, at least for relatively low density Hmode plasmas in AUG, SOLPS does not correctly predict divertor electron temperature and density. The plasma in the divertor calculated by the code must be too cold and dense, compared to the experiment. This, to a large degree, must be attributable to profile effects, with the code predicting too narrow ion and neutral fluxes near the target, resulting in the largest discrepancy with the experiment near the strike point position. Since a number of specific assumptions have been made in the code to model the selected H-mode plasma (see section 4), it is important to assess how the code output can be affected by varying certain parameters in the code that can be changed without violating the code integrity. One can also vary some other, input parameters, taken from the experiment, which may not be very well determined from the experiment (e.g., due to a large scatter in experimental points), or which can be affected by systematic shifts of the diagnostics.

The most severe, and obvious, discrepancy between the experiment and code simulations, is in the level of the peak $\mathrm{H}_{\alpha}$ radiation from the outer divertor which is significantly larger in the code (parameters at the inner divertor are usually affected by a fine balance between the available power supply and radiation losses, both due to impurities and the main working gas, and are therefore subjected to large swings which makes it more difficult to compare code 
output with experiment). Sensitivity studies of divertor conditions, through variation of assumptions and input parameters in the code, should therefore be aimed at bridging the large gap between experimental and simulated $\mathrm{H}_{\alpha}$ radiation profiles. Since the most likely explanation for the large discrepancy on the $\mathrm{H}_{\alpha}$ radiation, as well as some other discrepancies, is the too low divertor electron temperature predicted by the code (and hence, too high density), the peak outer target $T_{e}$ should be another important parameter to focus on in the sensitivity studies, with the aim of raising it substantially above values obtained earlier in the modelling.

As noted earlier, SOLPS cases with drifts take much longer to run and, in the case of this particular H-mode plasma, require the drifts to be switched off in the bulk of the core region (a deficiency which will, of course, be dealt with in the future). For the sensitivity studies, a reference non-drift case has therefore been chosen. The non-drift cases however are known to be affected by an unrealistic power sharing between divertors, where the inner divertor receives a disproportionally large fraction of the total power flux (but still, much less than $50 \%$ ). In the reference non-drift case therefore the input power into the grid was slightly raised, by about $10 \%$, above the experimental value, from 2.7 to $3.0 \mathrm{MW}$, to obtain more realistic conditions in the outer divertor where some parameters, mainly peak $\mathrm{H}_{\alpha}$ radiation and peak $T_{e}$ at the outer target, will be compared with the experiment. The power deposition profile for the non-drift case with 3.0 MW of input power is presented in Fig. 6, alongside other calculated and measured power deposition profiles. Apart from their impact on the power sharing between the two divertors, inclusion of drifts did not result in any significant variation of plasma parameters in the outer divertor, and drift effects could not explain the large discrepancies between simulated and measured parameters in the divertor. In all nondrift SOLPS cases described in this paper EIRENE was coupled B2, as in the drift case discussed above.

\section{(a) Variation of separatrix density}

The easiest way to raise the divertor $T_{e}$ and reduce $\mathrm{H}_{\alpha}$ radiation is to reduce the separatrix density, $n_{\text {sep }}$, which is a free parameter in the SOLPS calculations. As noted in Sec. 5, however, choosing the wrong $n_{\text {sep }}$ results in a poorer match between experimental and simulated temperature profiles. This is illustrated in Figure 10, where SOLPS fits for the case with $n_{\text {sep }}=1.3 \times 10^{19} \mathrm{~m}^{-3}$, reduced from the original, reference value of $1.6 \times 10^{19} \mathrm{~m}^{-3}$, are presented. Most of the experimental points on the $T_{e}$ profile just inside of the separatrix are now below the code values. The corresponding $\mathrm{H}_{\alpha}$ and CIII radiation profiles are shown in Figure 11 .

The large discrepancy in the peak $\mathrm{H}_{\alpha}$ radiation has not been eliminated, even though the ratio of peak simulated to experimental $\mathrm{H}_{\alpha}$ signals is reduced to 1.70 , compared with 2.12 in the reference case with the same input power, and the simulated $\mathrm{H}_{\alpha}$ radiation profile is still much narrower than the experimental one. Compared to a large discrepancy shown in Fig. 8, a satisfactory match between the peak values of the simulated and experimental CIII radiation profiles is now achieved, but the integrated simulated CIII emission is still substantially below that in the experiment. The peak $T_{e}$ at the outer target has only been raised to $18.8 \mathrm{eV}$, compared to the reference case with $T_{e, \max }=16.9 \mathrm{eV}$. Hence, the gains in reducing the large discrepancy between experimental and simulated $\mathrm{H}_{\alpha}$ signals and raising the peak $T_{e}$ at the outer target are not sufficient to drastically change the situation, while the quality of the match to the upstream experimental $T_{e}$ data has been substantially sacrificed. One should 
therefore conclude that reducing separatrix density cannot remove discrepancies between experiment and modelling.

\section{(b) Variation of electron and ion heat flux limiters}

Figure 12 shows the results of the variation of electron and ion parallel heat flux limiters in SOLPS runs, using peak $T_{e}$ at the outer target and the ratio of peak $\mathrm{H}_{\alpha}$ signals as figures of merit. The reference case, as explained in Sec. 4, has normalized electron and ion flux limiters 0.3 and 1.0, respectively. It has an $\mathrm{H}_{\alpha}$ ratio of 2.12 and a peak target $T_{e}$ of $16.9 \mathrm{eV}$. Both figures are clearly little affected by the variation in the flux limits. Conditions in the divertor must therefore be rather conservative, probably due to the fact that main gradients in the $T_{e}$ and $T_{i}$ distributions along the field lines occur in the divertor, where the plasma is much more collisional. Similar results have been earlier obtained in [5].

The choice of the ion flux limiter also had a very little impact on the $T_{i}$ in the divertor; further upstream, in the main SOL, however, the ion heat flux limiter was found to affect poloidal $T_{i}$ profiles quite significantly [24].

\section{(c) Variation of power sharing fractions}

The default power sharing used in the series of SOLPS runs described in this paper assumes equal power flows into the grid through ion and electron channels. The results of changing this assumption, by directing $75 \%$ of the input power to each of the channels, leaving only $25 \%$ for the other, are shown in Figure 13. As in the previous sub-section (b), the SOLPS solution for the divertor seems little affected by how exactly the power is shared between the channels at the core boundary of the grid. Energy exchange between ions and electrons, especially in the divertor where the plasma is cold and dense and the collision frequency is high, must be large enough to offset the effects of the initial difference between $T_{e}$ and $T_{i}$ in the core part of the grid.

\section{(d) Role of ballooning of transport coefficients}

By default, all SOLPS runs described here assume a dependence of all transport coefficients on the toroidal field of the type 1/B. This increases the 'natural ballooning' of the radial transport due to field line compression on the outer, low field side of the plasma, as was explained in Sec. 4. Figure 14 demonstrates how the alteration of the degree of ballooning, ranging from zero ballooning $\left(1 / \mathrm{B}^{0}\right.$ dependence) to a much increased ballooning $\left(1 / \mathrm{B}^{2}\right.$ dependence) impacts on the two figures of merit used here to characterize divertor conditions. Increase in the ballooning results in a modest rise in the peak outer divertor $T_{e}$ and a more substantial rise in the simulated ratio of the peak $\mathrm{H}_{\alpha}$ signals. Both effects are weak, however, and will not help to bridge the gap between experimental and simulated parameters in the outer divertor.

\section{(e) Variation of transport coefficients in the $S O L$}

SOLPS is normally run with transport coefficients varied poloidally in accordance with the $1 / \mathrm{B}^{\alpha}$ dependence, as mentioned above. The coefficients are also adjusted radially to match experimental profiles. There is an option, however, to scale the coefficients independently in the core + private region, main SOL (not including the divertor) and main divertor (part of the SOL) regions. Variations in transport coefficients must not, of course, lead to a strong violation of measured profiles. Only relatively small adjustments to the profiles are acceptable, in line with the scatter or potential systematic shifts in experimentally measured profiles. 
It is typically seen in the SOLPS output that increasing transport coefficients, especially $\mathrm{D}_{\perp}$, in the SOL makes the divertor effectively more closed by widening radial density/pressure profiles resulting in more efficient trapping of neutrals and leading to a drop in divertor $T_{e}$ and a rise in density. One may therefore expect that making the profiles sharper in the SOL will result in the increase in divertor $T_{e}$ and a reduction in the $\mathrm{H}_{\alpha}$ emission.

Figure 15 shows the modification in the density profile at the outer midplane as a result of multiplying all transport coefficients in the SOL (excluding the divertor) by factors 0.7 and 1.3. Such variations in the transport coefficients should be considered as maximum allowable, since more significant changes would severely violate consistency with the experimental density profile.

The influence of the variation of transport coefficients in the SOL on the peak $T_{e}$ at the outer target and the $\mathrm{H}_{\alpha}$ ratio is shown in Figure 16. As expected, the outer target $T_{e}$ is fairly strongly affected by the profile changes. At the same time, the $\mathrm{H}_{\alpha}$ ratio is much less affected. Since such changes in transport coefficients result in rather significant alteration of the simulated midplane density profile, by compromising its consistency with the experimental profile, there is a very limited scope in changing transport coefficients as a measure to resolve inconsistencies between experimental and simulated conditions in the divertor. A more efficient way of affecting simulated divertor conditions via changes in radial transport is considered in the next sub-section.

\section{(f) Variation of transport coefficients in divertor}

Figure 17 shows the result of a strong variation, by a factor 10 in each direction, of all transport coefficients in the divertor (the common, or SOL part of it) on the peak $T_{e}$ at the outer target and the $\mathrm{H}_{\alpha}$ ratio. The divertor-SOL region seems much more important than the private region, since most of the plasma density (or the ion saturation current, as seen on Langmuir probes) if found there. Despite a large variation in the coefficients, the profiles at the outer midplane (not shown here) were found to be very little affected by these changes. The peak outer target $T_{e}$ can be strongly reduced by increasing the transport in the divertor. However, the $\mathrm{H}_{\alpha}$ ratio is changed insignificantly. At the same time, reducing transport coefficients by the same factor results in much less significant changes in both the target $T_{e}$ and the $\mathrm{H}_{\alpha}$ ratio. The physics behind the sharp drop in the divertor $T_{e}$ following an increase in the transport coefficients must be the same as was mentioned in the previous sub-section: 'plugging' neutrals in the divertor by widening profiles results in more efficient re-circulation and ionization of neutrals. It is not clear, however, whether there is enough physical justification for a dramatic increase in the divertor transport coefficients compared to the ones in the main SOL. Besides, the sharp reduction in the simulated peak outer target $T_{e}$ would be in a clear disagreement with both direct and indirect experimental evidence for this quantity.

\section{(g) Variation of He content}

Following a series of unsuccessful attempts to reduce inconsistencies between simulated and measured divertor conditions (as manifested mainly by very small impact on the high $\mathrm{H}_{\alpha}$ ratio) by varying assumptions about the plasma transport, some other ways to achieve the same goal have been attempted. One of them was to change the plasma mix by adding a certain amount of helium to the ion component. From the available experimental data, one cannot rule out a possible helium content of up to $20 \%$, with the remainder being mostly deuterium, and a very small fraction of hydrogen. The two SOLPS cases, one without helium and the other with $20 \%$ of helium content were compared, and the results showed almost no difference between midplane as well as divertor profiles. The only exception was higher 
midplane ion temperature, attributable to smaller number of ions (since helium ions are doubly charged) for the same amount of input power into the grid through the ion channel.

\section{(h) Variation of sheath boundary conditions at the targets}

Strict Bohm boundary conditions at the targets, requiring the Mach number of the parallel ion flow to be equal to 1 at each target, are normally used in SOLPS. To test the effect of relaxing these sheath boundary conditions, a separate SOLPS case, by allowing the Mach number to exceed 1 but not allowing it to fall below this value, has been run. A significantly higher ion velocity at the outer target may result in a drop in the plasma density there, owing to the conservation of the parallel stress $\left(n T_{e}+n T_{i}+n m_{i} V_{i \|}^{2}\right)$.

No significant changes due to the relaxation of the boundary conditions have been detected in the code output. Compared with the reference case, the Mach number at the inner target on the first ring outside of the separatrix has increased from 1 to 1.18, and at the outer target from 1 to 1.16. Electron density on these rings has dropped by factors 1.18 and 1.13, respectively. In the outer SOL, the rise in the Mach number at the inner target was more significant, up to $\approx 1.2$, but it was rather insignificant at the outer target, being below 1.05.

\section{(i) Use of a more realistic neutral description in the vacuum vessel}

In all SOLPS cases a simplified wall/pumping description was adopted, which assumed $100 \%$ recycling at the walls, ignored the large drop in measured neutral flux between pressure gauges in the sub-divertor and pumping regions, and ignored the experimental removal rate of neutrals by the pumping system. These shortcomings of the simplified neutral model have been dealt with in a dedicated series of runs aimed at re-constructing experimental features of the in-vessel neutral transport.

The calculated neutral removal rate by the cryo-pump in the experiment was equivalent to $\approx$ $275 \mathrm{~A}$, by far exceeding neutrals input into the grid of $\approx 80$ A by the NBI source. Hence, one has to take into account outgassing of the walls during the discharge. The results of a detailed particle balance calculations are presented in Fig. 18. They include all known particle sources (gas puffing valves and the NBI source) and sinks (by cryo, turbo and NBI pumps), as well as the evolution of the plasma density content (almost unchanged between 2.1 and $4.5 \mathrm{~s}$ ). Due to a substantial excess of the particle removal by pumps over particle sources, the curve labeled 'Net balance' in Fig. 18 has a negative slope after $\approx 2 \mathrm{~s}$ of the discharge time, when the initial gas puffing was switched off, and up to the start of a heavy gas puffing at $4.5 \mathrm{~s}$. This implies that during this discharge phase the wall was serving as a net source of particles. Towards the end of this phase, the net calculated particle balance becomes negative after $\approx 3.5 \mathrm{~s}$, indicating that the wall has become the net source of particles in the system. The number of particles released from the wall between 3.5 and $4.5 \mathrm{~s}$ is equal to the whole plasma particle content, as one can see from Fig. 18.

It has also been established earlier in AUG that the real removal rate of neutrals from the pumping region is increased by the neutral by-pass leaks from this region into the plasma volume through gaps in the divertor structure. These leaks can be characterized by a removal rate of $\approx 100 \mathrm{~m}^{3} \mathrm{~s}^{-1}$, thus making the total effective removal rate of neutrals from the pumping region $\approx 200 \mathrm{~m}^{3} \mathrm{~s}^{-1}$, of which, of course, the actual pumping was only half of that value. Taking into account the measurements of the ionization pressure gauges, the removal rate of $200 \mathrm{~m}^{3} \mathrm{~s}^{-1}$ is translated into an equivalent particle removal rate of $\approx 450 \mathrm{~A}$. The combined 
effect of the wall outgassing and by-pass leaks have been modelled in Eirene by using a uniform gas puff rate into the plasma equivalent to $450-80=370 \mathrm{~A}$.

The measured large drop of neutral flux from the sub-divertor region to the pumping region, as has already been described in Sec. 3, was approximately matched in the SOLPS modelling by the introduction of a baffle in Eirene (see Fig. 3b), roughly accounting for blockages for the neutral exchange between sub-divertor and pumping regions by various divertor and wall structures.

The results of SOLPS runs with a more refined neutral description, with respect to the divertor conditions, have been almost the same as those with a simplified neutral model. The combined effect of the gas puffing and increased pumping was a drop in the sub-divertor neutral flux by $14 \%$. The neutral density there was still $37 \%$ higher than in the experiment. The calculated neutral flux at the outer midplane was $67 \%$ of the measured value. (The midplane pressure flux $2.5 \times 10^{20} \mathrm{~m}^{-2} \mathrm{~s}^{-1}$, measured by the pressure gauge 17 shown in Fig. $3 \mathrm{~b}$, cannot be directly compared with SOLPS results. The code assumes toroidal symmetry, while the gauge is mounted on the ICRH antenna, which is protected by the two limiters, and there are 4 such antennas in AUG. Readings of the gauges 14 and 15, which measure neutral particle flux coming from other ports, both show fluxes equal to $0.8 \times 10^{20} \mathrm{~m}^{-2} \mathrm{~s}^{-1}$, much lower than the gauge 17. A more relevant figure of merit for a toroidally symmetric SOLPS simulation could be the average of these three gauges, $\approx 1.4 \times 10^{20} \mathrm{~m}^{-2} \mathrm{~s}^{-1}$ ). Midplane profiles of $n_{e}, T_{e}$ and $T_{i}$ were almost the same as in the runs with the simplified neutral model, for unchanged transport coefficients. This indicates a good balance between extra sources of neutrals, originating from the wall, and sinks due to the increased pumping. A comparison between outer target $T_{e}$ profiles and the $\mathrm{H}_{\alpha}$ emission profiles from the divertor (both inner and outer targets are covered) obtained in the SOLPS runs with the simplified and more detailed neutral models are presented in figure 19. Almost no difference is seen in the profiles of the $\mathrm{H}_{\alpha}$ emission at the outer target, while the peak target $T_{e}$ is lower by $\approx 2 \mathrm{eV}$ for the case with the detailed neutral model. A weak influence of the choice of a pumping model on divertor parameters has been found earlier in [24].

\section{(j) Choice of a model for chemical sputtering}

In all of the SOLPS cases presented here, constant physical and chemical sputtering rates have been used. It is already well established, however, that the chemical sputtering rate, which was a dominant source of impurities in the cases, strongly depends on the surface temperature of the target plates and on the impinging plasma flux, both ion and neutral (see e.g. [25]). Regarding the flux dependence, chemical sputtering rate decreases as the flux is increased above a certain level. This level was exceeded by a large margin everywhere along the outer target except for the extreme far SOL region. For the same integrated carbon influx from the targets, one should therefore expect fewer sputtered atoms per unit deuterium flux near the strike point than further away from it. An assumption of the constant sputtering rate could have therefore adversely affected the SOLPS solutions by causing excessively large sputtered fluxes near the strike point. This must have caused extra, unrealistic cooling of the plasma near the strike point, leading to an increase in the plasma density there and hence, $\mathrm{H}_{\alpha}$ radiation.

In the present modelling, it was not possible to directly check the influence of the fluxdependent chemical sputtering rate on the SOLPS solution near the strike point. Apart from the flux-dependence, there is also a strong dependence of the sputtering rate on the surface temperature. Due to various technical problems, the correct dependence for the chemical 
sputtering yield has not yet been implemented in the coupled (EIRENE + B2 codes) selfconsistent mode. As an alternative, the dependence of the code solutions near the strike point on the magnitude of the chemical sputtering rate for the whole target was checked. In an extreme case, both physical and chemical sputtering have been completely switched off, in order to remove a contribution of volumetric impurity radiation sources to the formation of dense and cold plasma just outside of the separatrix near the outer target. Nevertheless, only limited increase, from 16.9 to $19.0 \mathrm{eV}$, in the maximum $T_{e}$ at the outer target was observed in the simulations. The total radiated power inside of the grid has dropped from 1.19 to 0.87 $\mathrm{MW}$, as a result of a combination of the complete removal of impurity radiation (which stood at $0.49 \mathrm{MW}$ ) and the rise in the deuterium radiation (from 0.70 to $0.87 \mathrm{MW}$ ). The simulated peak $\mathrm{H}_{\alpha}$ radiation at the outer target was increased by $15 \%$. On the basis of this comparison one may conclude that the amount of impurity radiation inside of the flux tube bordering the separatrix was not a critical parameter in establishing the highly peaked $\mathrm{H}_{\alpha}$ radiation profile at the outer target. Rather, it must mainly be a consequence of the particle balance, with amplification of the peak ion flux to the target by ionization of neutrals, as a local increase in plasma density creates a feedback loop where the more efficient ionization of neutrals in this narrow layer further reduces the local plasma temperature and hence, increases density.

\section{(k) Variation of prompt reflection coefficient of neutrals from the target}

The ratio of peak $\mathrm{H}_{\alpha}$ emissions (simulated over experimental) was found to be one of the most resilient figures in the sensitivity studies. The width of the simulated $\mathrm{H}_{\alpha}$ emission profiles, much narrower than that in the experiment, was also little affected by all the changes made to the SOLPS runs which don't contradict to main experimental profiles and other parameters that must be matched by the code. The narrowness of the $\mathrm{H}_{\alpha}$ emission profiles and the large drop in the target $T_{e}$ at the position of the maximum density points to the tendency for the plasma, as well as neutrals, to concentrate near the separatrix position more than is observed in the experiment. An attempt was made to spread the plasma-neutral mix more widely radially, by making neutrals, rather than charged particles as in some cases described earlier, more mobile radially. The probability of prompt reflection of neutrals from the target was increased by factor 2 in Eirene to have more fast atoms near the target rather than slow molecules.

The above modification to Eirene led to an increase of the peak target $T_{e}$ and a small reduction in the level of the $\mathrm{H}_{\alpha}$ radiation, while the total radiated power inside of the computational grid dropped. After the radiated power was restored by raising chemical sputtering rate from 1.4 to $2.2 \%$, the $\mathrm{H}_{\alpha}$ emission profile became almost undistinguishable from that in the reference case, but the peak Te at the outer target increased from 16.9 to 22.5 $\mathrm{eV}$. The ratio of the average density of atoms to that of molecules in the outer divertor increased from 0.61 to 0.90 , in line with expectations. It is not clear why these changes have not resulted in a wider $\mathrm{H}_{\alpha}$ emission profile. This could be caused by the fact that the energy reflection coefficient from the surface ( $\mathrm{D}$ on $\mathrm{C}$ ) decreases with incident energy of energies above $10 \mathrm{eV}$, for almost all angles of incidence, or by some unexpected consequences of increased radiation and carbon impurity concentration.

\section{(l) Large reduction of separatrix density and input power}

As discussed earlier, owing to lower collisionality upstream of the targets, a substantial fraction of supra-thermal ions and electrons in the divertor is expected. It is likely (and will be a subject of further studies) that their contribution to SOLPS (B2) equations cannot be accommodated by only varying flux limits for ion and electron parallel heat fluxes. An attempt was therefore made to try to model only the 'thermal' component of the plasma, by 
disregarding a certain fraction of the measured electron density as a 'supra-thermal' component which contributes to the power deposition at the target plate but doesn't contribute much to the plasma density in the divertor. One may then, in the modelling, choose a much lower effective separatrix density and a much lower input power for the 'thermal' component. In the case described below, the input power into the grid was reduced to 1.8 MW, and separarix density - to $9 \times 10^{18} \mathrm{~m}^{-3}$, in an attempt to match the Langmuir probe profiles at the outer target and the experimental $\mathrm{H}_{\alpha}$ radiation.

With the above input parameters, target profiles of electron density and temperature measured by the Langmuir probes could indeed be very well matched. The peak $\mathrm{H}_{\alpha}$ radiation was close to its value in the experiment, but the integral $\mathrm{H}_{\alpha}$ radiation was by a factor 2 below the experiment one due to profile effects. The unrealistic peaking of the $\mathrm{H}_{\alpha}$ radiation signal could therefore not be eliminated. The simulated sub-divertor pressure was below the experimentally measured, by $\approx 40 \%$. The largest disagreement with the experiment was in the amount of the radiated power in the divertor, with the simulated signal being only $\approx 50 \%$ of the experimental one. However, in order to achieve even this, much reduced, level of radiation, chemical sputtering had to be almost doubled, to 3\%. This, on the other hand, brought the carbon density in the core part of the grid to $\approx 1 \%$, in line with the experiment. Further increase in the chemical sputtering rate was not possible, since it would not only result in a much higher carbon concentrations than in the experiment, but would also reduce target $T_{e}$, below the Langmuir probe data. Summarizing, an attempt to ignore a fraction of the measured upstream density in the SOLPS modelling as being attributed to the 'supra-thermal' component has not resulted in a satisfactory match with a number of experimental parameters, with the most severe disagreement being in the radiation power inside of the computational grid.

\section{Comments on measured and calculated ion target fluxes and $H_{\alpha}$ emission profiles}

Repeated failures to reduce substantially the peak $\mathrm{H}_{\alpha}$ signal simulated in various SOLPS runs described in the previous section might put into question the validity of the simulation of this spectroscopic signal in the code. Namely, one may suspect this simulated signal of being greatly overestimated, since it stays persistently above the corresponding experimental signal. There is a proof, however, that this is not the case and that the simulated $\mathrm{H}_{\alpha}$ signal is not internally inconsistent with the ion flux to the target, at least, it cannot be regarded as being excessively large. Similarly, experimental ion flux to the target is largely consistent with the measured $\mathrm{H}_{\alpha}$ emission. This is demonstrated in figure 20, where the $\mathrm{H}_{\alpha}$ emission measured in photons per unit area and per steradian has been converted into an ion flux using the usual figure of merit of the emission multiplied by $4 \pi \times 20$.

The case with drifts and input power flux of $2.7 \mathrm{MW}$ was used to plot the data in Fig. 20 . Experimental data for $j_{s a t} / e$ compares very well with the corresponding signal derived from the $\mathrm{H}_{\alpha}$ emission. The measured $j_{\text {sat }}$ is most likely to be underestimated, owing to an apparent underestimate of $T_{e}$ by Langmuir probes, as discussed in Sec. 5. As a result, the real saturation in the ion current has probably not been achieved. The calculated ion flux to the target is very close to $H_{\alpha} \times 4 \pi \times 20$ far outside of the separatrix, but exceeds it by a large margin near its peak value. The simulated signal is substantially above the measured one, as discussed earlier, and has a narrower profile. At any rate, the simulated $\mathrm{H}_{\alpha}$ emission cannot 
be regarded as being unreasonably large, given the magnitude of the simulated ion flux to the target.

The large, by an order of magnitude, difference between calculated ion flux to the target and the experimental $j_{\text {sat }} / e$ near their peak values, cannot be explained only by an underestimate in $j_{\text {sat }}$. An additional reason for this discrepancy must be a large overestimate of electron density by SOLPS, caused by an unrealistically low calculated $T_{e}$.

\section{Summary}

The SOLPS code has been used for steady-state modelling of inter-ELM plasma of one of the best diagnosed low density ( $\approx 60 \%$ of the Greenwald limit) H-mode shots in ASDEX Upgrade. Reliable edge density and temperature profiles have been measured in the experiment, covering the pedestal region and stretching far into the SOL. A number of divertor parameters and profiles have also been obtained. This greatly constrained the modelling regarding the possible choice of arbitrary input parameters in the code thereby enabling one to test consistency of code solutions with the experiment.

Measured midplane $n_{e}, T_{e}$ and $T_{i}$ profiles have been fitted by adjusting (radially variable) transport coefficients in the code. Given the constraint imposed on the code solutions by the amount of the input power into the grid, one of the critical input parameters in the modelling, the value of the separatrix density $n_{\text {sep }}$, could be established. This parameter is usually very difficult to determine in experiment with sufficient accuracy. A further constraint imposed by the radiated power inside the grid evaluated from the experiment determines the main divertor parameters: electron density and temperature, as well as several parameters that can be directly compared with the experiment. The code solutions were found to be broadly in agreement with many experimental parameters in the divertor. It was also possible to reconstruct neutral fluxes measured by in-vessel ionization gauges, with accuracy of $\approx 40 \%$, by using a rather sophisticated neutral model in Eirene (neutral part of SOLPS), which required certain alterations in the in-vessel structures close to the divertor and an assumption (supported by the experiment) of a strong wall outgassing during the discharge which was modelled by poloidally uniform gas puffing.

Measured parameters in the divertor had some internal inconsistencies, arising mainly due to somewhat contradictory data supplied by target Langmuir probes. The whole set of experimental data in the divertor has nevertheless provided sufficient information to identify one critical issue with the SOLPS solutions. Namely, the code predicts too dense and cold (low $T_{e}$ ) plasma in the divertor, leading to some inconsistencies between measured and simulated parameters. In particular, the peak value of the simulated $\mathrm{H}_{\alpha}$ radiation profile in the outer divertor is substantially, by factor $\sim 2$, above its experimental value, and the simulated profile is narrower than in the experiment. The integrated $\mathrm{H}_{\alpha}$ emission from the outer divertor, on the other hand, is quite close to the experimental one. The code also tends to overestimate the sub-divertor neutral flux and underestimate CIII emission at the target, as well as carbon impurity content in the core edge region, which could be directly compared with the experiment. All these inconsistencies imply that the simulated plasma in the outer divertor is denser and cooler than in the experiment, with the deviation from real experimental conditions becoming especially pronounced near the strike point position. Similar results have been earlier observed at JET, where in H-mode plasmas target Langmuir probe data could not be matched by EDGE2D modelling inside of a narrow layer a few 
millimeters around the separatrix [26]. The simulated target density was considerably higher, and the electron temperature lower, than the experimental data.

The discrepancy of a factor 2 between peak values of the simulated and experimental $\mathrm{H}_{\alpha}$ profiles should make a profound effect on the predicted density and temperature near the strike point. Indeed, assuming that the local $\mathrm{H}_{\alpha}$ emission is proportional to the ion flux to the target, and using a simple relation between the ion flux and local plasma parameters, implies: $H_{\alpha} \sim n_{e} \sqrt{T_{e, i}}$. For fixed pressure $n_{e} T_{e, i}$ (which should be proportional to the pressure upstream), this relation yields a rather steep dependence of the target electron/ion temperature on the $\mathrm{H}_{\alpha}$ radiation: $T_{e, i} \sim H_{\alpha}^{-2}$. Hence, an overestimate of the peak $\mathrm{H}_{\alpha}$ signal by factor 2 can potentially result in an underestimate of target plasma temperature(s) by a factor 4 .

Extensive sensitivity studies of the influence of variation in input parameters and assumptions of the code on the modelled divertor conditions have been conducted. In particular, attempts were made to influence code solutions via energy balance by e.g. varying ion and electron parallel heat flux limits, or removing impurity radiation in order to increase the peak target $T_{e}$. None of the measures taken was successful enough to remove a discrepancy with the measured $\mathrm{H}_{\alpha}$ radiation profile in the outer divertor which remained substantially wider, but with a lower peak value than in the code. Two possible explanations for this discrepancy can be given: one related to some missing reactions/processes in the Eirene that could e.g. increase the energy of neutrals and thus prevent the tendency for the neutral-plasma mix to congregate excessively around the strike position, the other - to kinetic effects related to low collisionality.

Although the modelled peak $T_{e}$ at the outer target, as well as the whole $T_{e}$ profile, is broadly in agreement with the target Langmuir probe data, there is a compelling indirect evidence to suggest that both modelled and experimental electron temperatures are underestimated. Apart from inconsistencies mentioned above, there exists a large discrepancy between the power fluxes to the outer target measured by the IR camera and deduced from Langmuir probes. The probes severely underestimate the target power flux, which is also inconsistent with the power balance considerations.

Due to low density and relatively high input power (of $\approx 5 \mathrm{MW}$ ), the separatrix $T_{e}$ predicted by SOLPS was high, above $100 \mathrm{eV}$. Under these conditions one can expect ion and electron kinetic effects to play a significant role in the parallel transport. In particular, the presence of energetic tails in the electron distribution function or even a bi-Maxwellian distribution may be expected. This could have affected the $T_{e}$ measurements by the Langmuir probes, where the real ion saturation current has probably not been reached (and may have been impossible to reach even by a substantial increase in the biasing voltage due to an expected strong rise in the secondary electron emission). Significant fractions of supra-thermal ion and/or electron components in the divertor might lead to an increase in the averaged plasma temperature at the target, thereby reducing the plasma density. An attempt was made to consider a large fraction (almost 44\%) of the plasma upstream as being 'supra-thermal', and to model only the remaining 'thermal' component with SOLPS. The results, however, were negative since, apart from the expected effect of reducing simulated $\mathrm{H}_{\alpha}$ radiation, some new discrepancies have occurred. The largest was a strong reduction in the radiated power in the divertor, which could not be raised significantly even by an almost two-fold increase in the chemical sputtering rate (to 3\%). As a result, the calculated carbon impurity concentrations at the core 
edge of the numerical grid were beginning to exceed those measured in the experiment, while $\approx 50 \%$ of the experimental radiated power was still unaccounted for.

There have been earlier indications that SOLPS, as well as other edge codes, may overestimate the density in the divertor [27], at least in plasmas with not very high densities. It is not clear at present whether this originates from an intrinsic problem of collisional codes missing some important non-collisional effects, which are impossible to describe by e.g. choosing the appropriate flux limits, or from inadequate treatment of neutrals and their interactions with the charged particles in the code, or both. The planned SOLPS modelling of more collisional AUG plasmas, as well as an extention of the list of reactions included in EIRENE, should answer some of these questions.

\section{Acknowledgements}

The authors wish to acknowledge Dr. A.S. Kukushkin for useful discussions.

\section{References}

[1] Schneider R, Reiter D, Zehrfeld H P, Braams B, Baelmans M, et al., J. Nucl. Mater. 196-198 (1992) 810.

[2] Reiter D, J. Nucl. Mater. 196-198 (1992) 80.

[3] Coster D, Bonnin X, Borrass K, Bosch H-S, Braams B, et al., in Proc. of the $18^{\text {th }}$ IAEA Conference, Fusion Energy, Sorrento, Italy, October 2000, (CD-ROM), paper IAEA-CN77/EXP5/32, Vienna, 2000, IAEA.

[4] Coster D, Bonnin X, Braams B, Bürbaumer H, Kaveeva E, et al., in Proc. of the $19^{\text {th }}$ IAEA Conference, Fusion Energy, Lyon, France, October 2002, (CD-ROM), ages IAEA-CN94/TH/P2-13, Vienna, 2002, IAEA.

[5] Coster D P, Bonnin X, Braams B, Reiter D, Schneider R, et al., Phys. Scr. T 108 (2004) 7.

[6] Kukushkin A S, Pacher H D, Plasma Phys. Control. Fusion 44 (2002) 931.

[7] Schneider R, Coster D P, Kallenbach A, Borrass K, Bosch H S, et al., 'Test of the Predictive Capability of B2-EIRENE on ASDEX Upgrade', F1-CN-69/THP2/05.

[8] Schneider R, Coster D P, Neuhauser J, Bosch H S, Fuchs J C, et al., 'B2-Eirene modelling of the CDH mode in ASDEX Upgrade', http://www.rzg.mpg.de/ dpc/rfs_poster_eps95/paper/eps95/eps95.html

[9] Taroni A, Corrigan G, Radford H, Simonini R, Spence J, et al., Contrib. Plasma Phys. 32 (1992) 438.

[10] Simonini R, Corrigan G, Radford G, Spence J, Taroni A, Contrib. Plasma Phys. 34 (1994) 368.

[11] Taroni A, Corrigan G, Radford G, Simonini R, Spence J, et al., Technical Report JETC94089, JET Joint Undertaking, Abingdon, Oxfordshire, OX14 3EA, UK, 1994.

[12] Rognlien T, Brown P, Campbell R, Kaiser T, Knoll D, et al., Contrib. Plasma Phys. 34 (1994) 362, Fourth Workshop on Plasma Edge Theory, October 1993, Varenna, Italy.

[13] Stangeby P C, in The Boundary of Magnetic Fusion Devices, IOP Publishing, Bristol (2000), p.656.

[14] Radford G, Contrib. Plasma Phys. 32 (1992) 297.

[15] Fundamenski W, Plasma Phys. Control. Fusion 47 (2005) R163-R208.

[16] Eckstein W, Coomputer Simulation of Ion-Solid Interactions in Springer Series in Materials Science, Vol.10, Springer, Berlin, 1991. 
[17] Eckstein W, Heifetz D B, J. Nucl. Mater. 145-147 (1987) 332. ('Data Sets for Hydrogen Reflection and their Use in Neutral Transport Calculations')

[18] Horton L D, Chankin A V, Chen Y P, Conway G D, Coster D P, et al., Nucl. Fusion 45 (2005) 856.

[19] Garcia O E, Horacek J, Pitts R A, et al., Plasma Phys. Control. Fusion 48 (2006) L1-L10.

[20] Chankin A V, J. Nucl. Mater. 241-243 (1997) 199.

[21] Pitts R A, Andrew P, Bonnin X, et al., J. Nucl. Mater 337-339 (2005) 146.

[22] Kirnev G S, Corrigan G, Coster D, Erents S K, Fundamenski W, Matthews G F, Pitts R A, J. Nucl. Mater 337-339 (2005) 271.

[23] Fundamenski W, Pitts R A, Matthews G F, Riccardo V, Sipilä S and JET EFDA Contributors, Nucl. Fusion 45 (2005) 950.

[24] Coster D P, Bonnin X, Corrigan G, Kirnev G S, Matthews G, Spence J, J. Nucl. Mater. 337-339 (2005) 366.

[25] Roth J, Preuss R., Bohmeyer W, Brezinsek S, Cambe A. et al., Nucl. Fusion 44 (2004) L21-L25.

[26] Kallenbach A, Andrew Y, Beurskens M, Corrigan G, Eich T, et al., Plasma Phys. Control. Fusion 46 (2004) 431.

[27] Neuhauser J, Kallenbach A, private communication. 


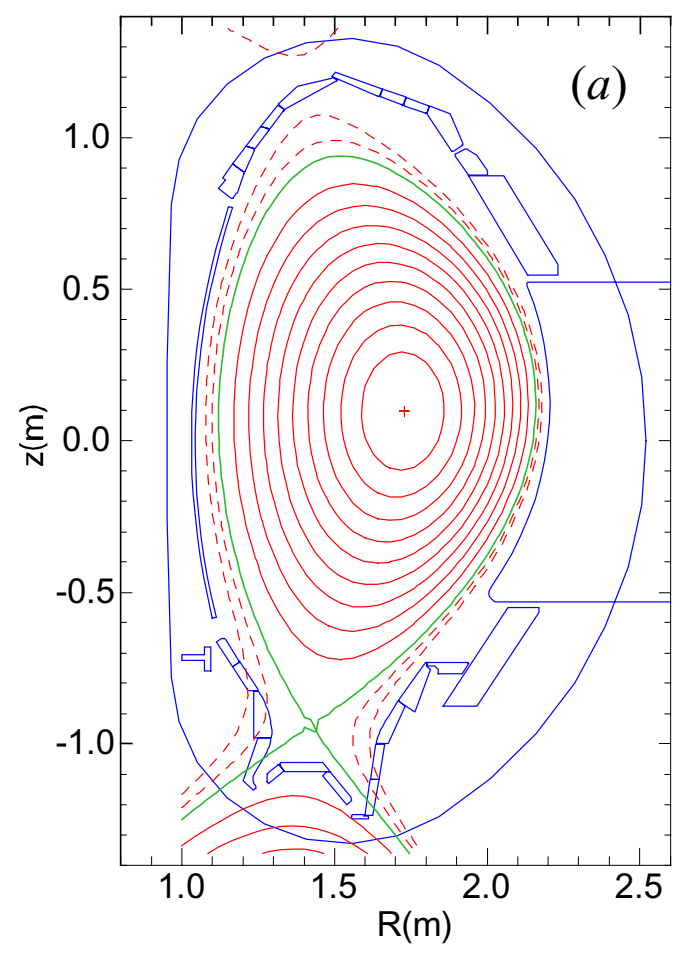

AUG Flux: $\Psi_{\mathrm{pol}}$

Shot: 17151

Time: 4.130

AUGD : FPP(1)

From FPG

$\mathrm{I}_{\mathrm{p}}: 0.80 \mathrm{MA}$

$\beta_{p}: 1.37$

$\mathrm{I}_{\mathrm{i}}: 0.87$

$\delta_{\wedge}: 0.228$

$\delta_{v}: 0.416$

$\kappa: 1.73$

$\mathrm{z}_{\mathrm{c}}: 0.083 \mathrm{~m}$

$\mathrm{R}_{\mathrm{c}}: 1.696 \mathrm{~m}$

$a_{\text {hor }}: 0.509 \mathrm{~m}$

$\mathrm{z}_{\mathrm{gi}}:-1.048 \mathrm{~m}$

$\mathrm{z}_{\mathrm{ga}}:-1.327 \mathrm{~m}$

$q_{95}:-5.01$

$\mathrm{R}_{\text {aus }}: 2.158 \mathrm{~m}$

vol : $13.88 \mathrm{~m}^{3}$

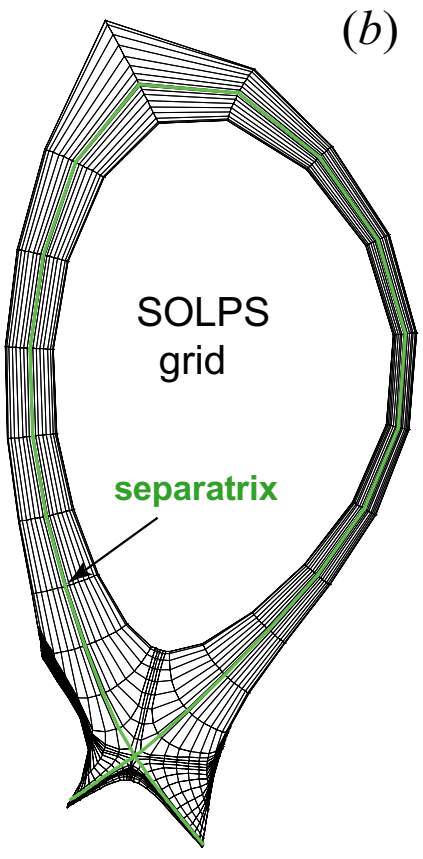

Figure 1. Magnetic configuration (a) and SOLPS grid (b) of the H-mode shot \#17151.
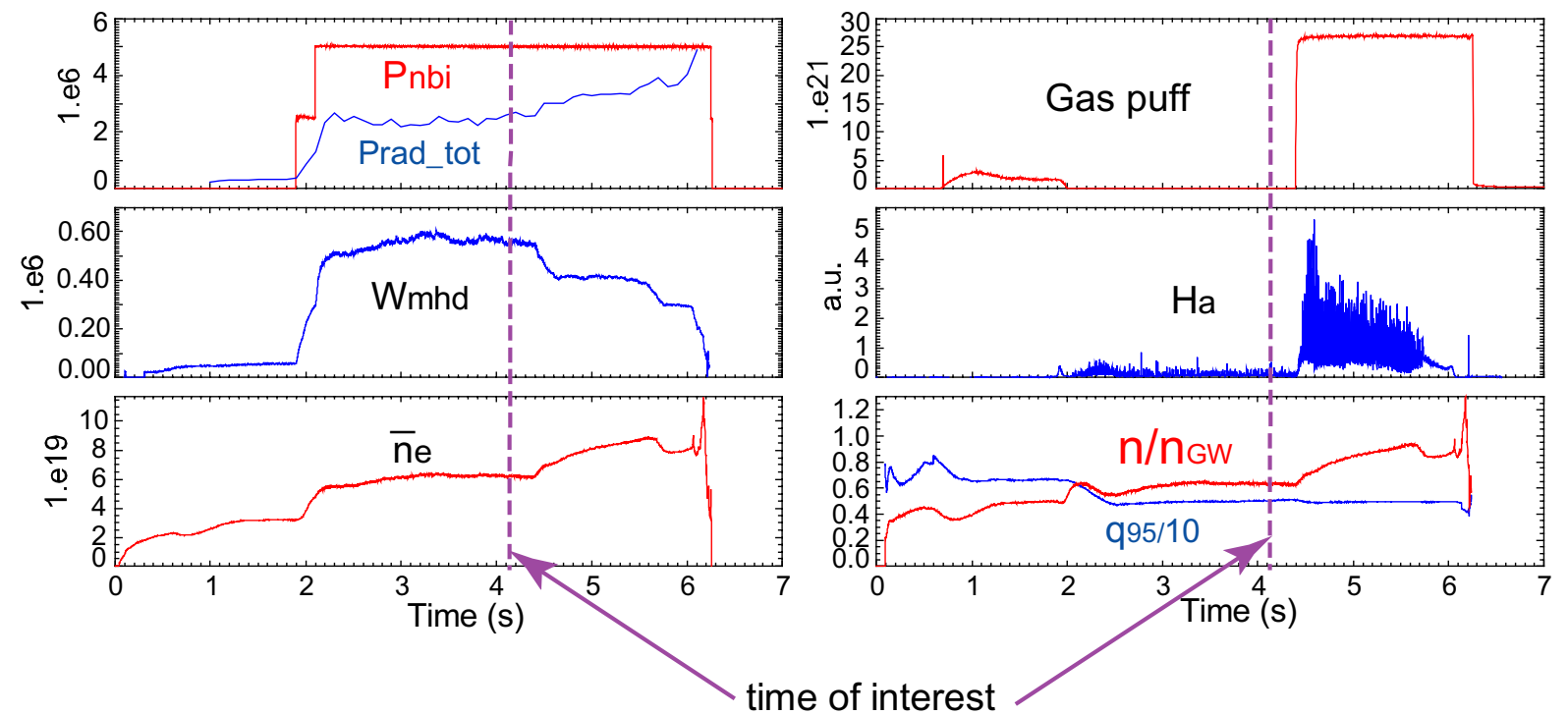

Figure 2. Experimental time traces of NBI power (Pnbi), total radiated power (Prad_tot), total plasma energy from MHD calculations (Wmhd), line averaged electron density $\bar{n}_{e}$, gas puffing rate, $H_{\alpha}$ radiation intensity $(\mathrm{Ha})$, Greenwald density fraction $\left(n / n_{G W}\right)$, and safety factor at the surface representing $95 \%$ of poloidal magnetic flux $\left(q_{95}\right)$. The 'time of interest' marker refers to the time $4.13 \mathrm{~s}$ of the discharge. 

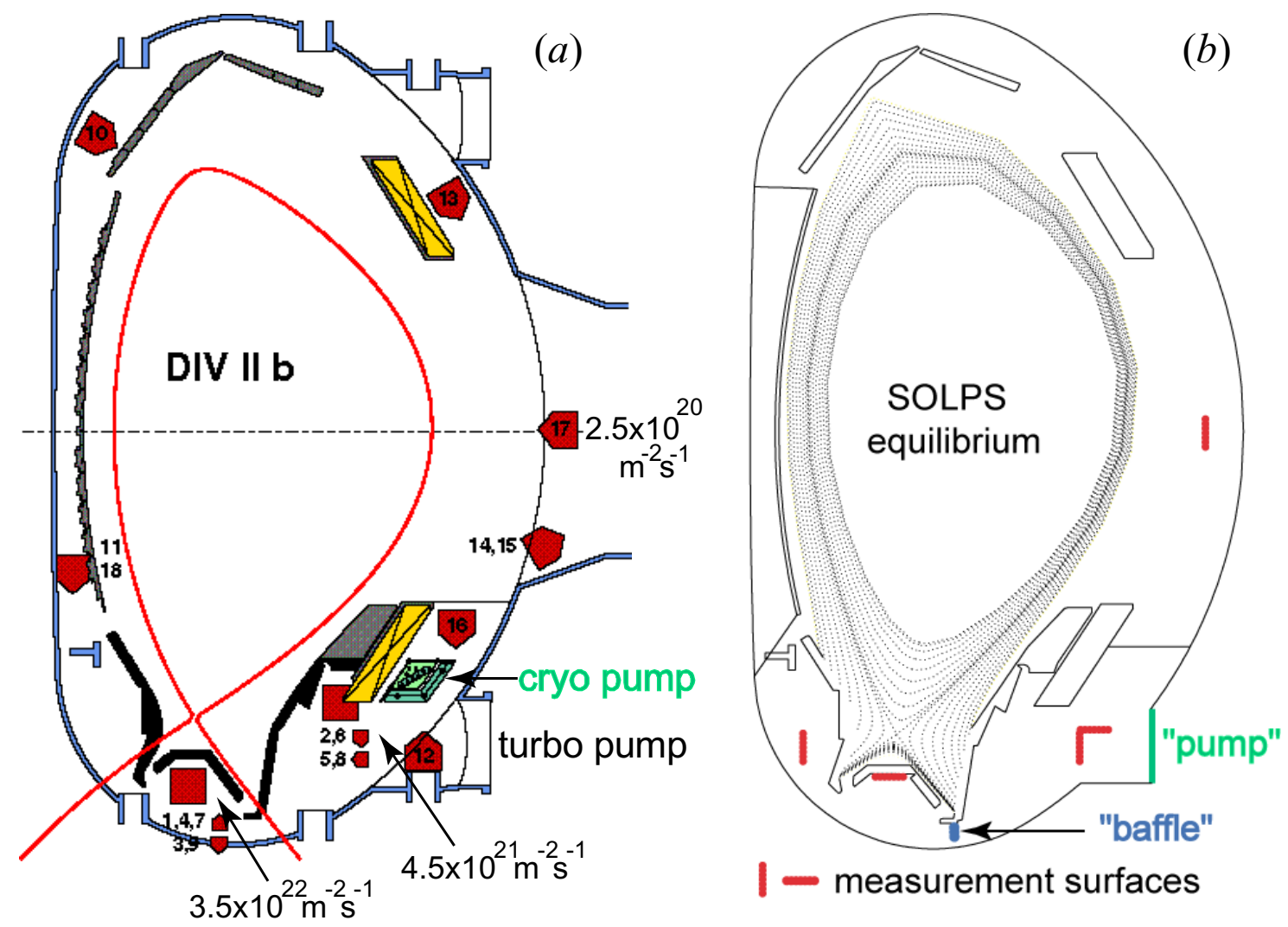

Figure 3. Position of ionization pressure gauges in AUG, alongside some divertor and wall structures (a), and SOLPS equilibrium with transparent 'measurement surfaces', schematic 'pump' and 'baffle' introduced in Eirene (see text below for details) (b). Positions of entrance slits for neutral fluxes into the gauges are marked by the tips of short red arrows. Figures indicate total measured neutral fluxes below the roof buffle (under the divertor), in the pumping region behind the outer divertor plate, and at the outer midplane. Top divertor structures shown in the figures have not been updated (hence, some difference with Fig. 1). 


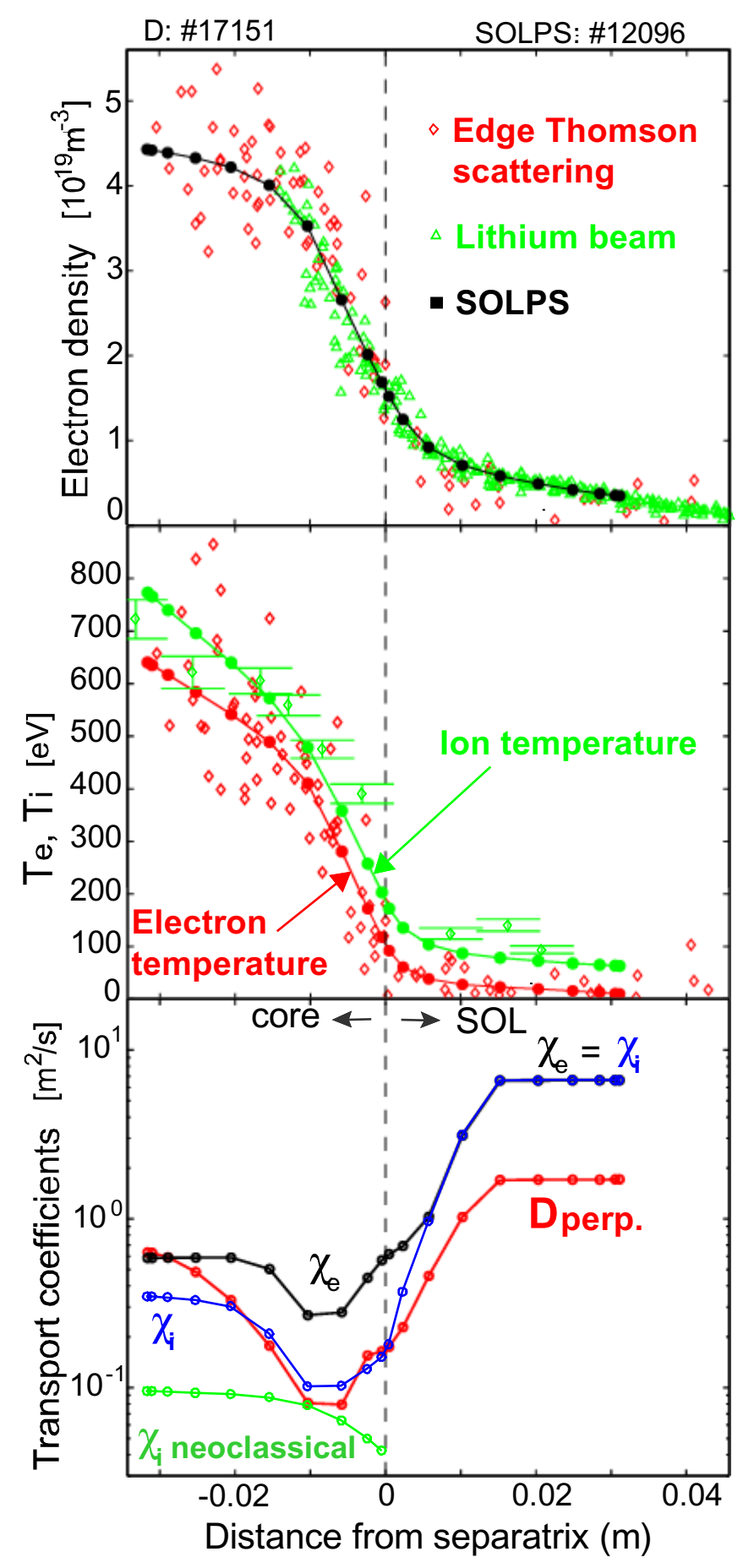

Figure 4. Profiles of electron density, electron and ion temperatures, together with SOLPS fits to the data and transport coefficients used in the calculations, for the selected H-mode shot \#17151. 

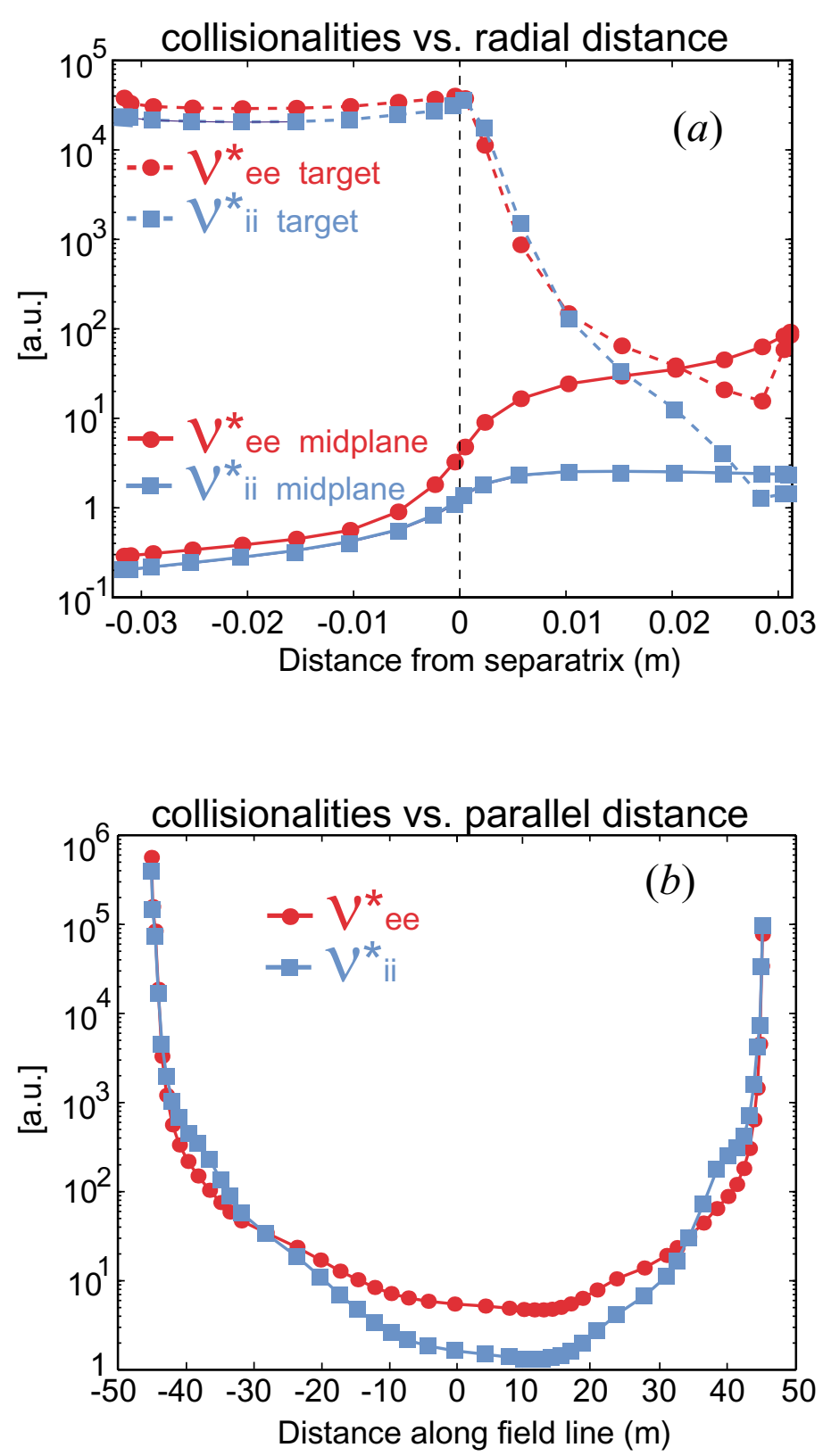

Figure 5. Electron and ion collisionality parameters for the case shown in Fig. 4, along radial distance at the outer midplane position, and along the outer divertor target, with the positions being mapped to the outer midplane (a), and along parallel distance from the inner target (negative distances) to the outer (positive distances) along the first SOL ring flux tube, (b). 


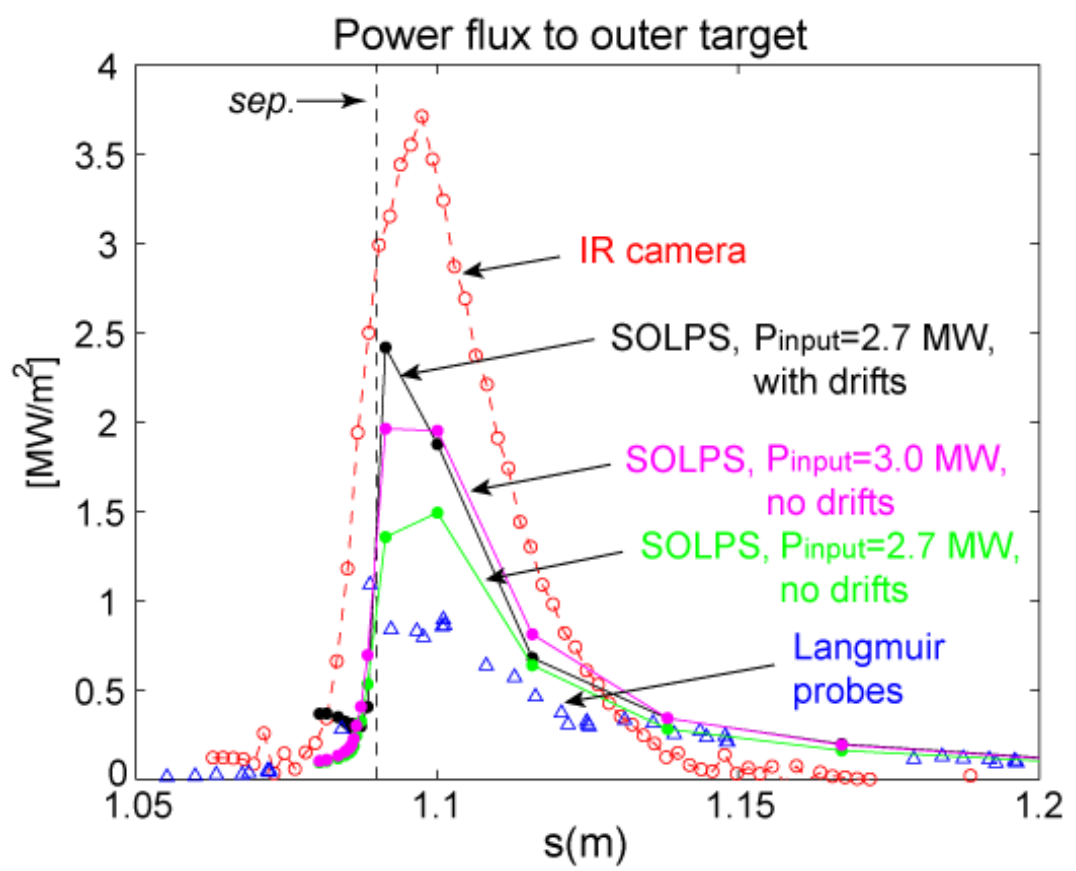

Figure 6. Outer target profiles of power deposition from IR camera, Langmuir probes, and calculated by SOLPS using 2.7 MW input power into the grid with drifts included, and 2.7 $M W$ and 3.0 MW of input power in calculations without drifts. Profiles are plotted against the coordinate 's' along the target.

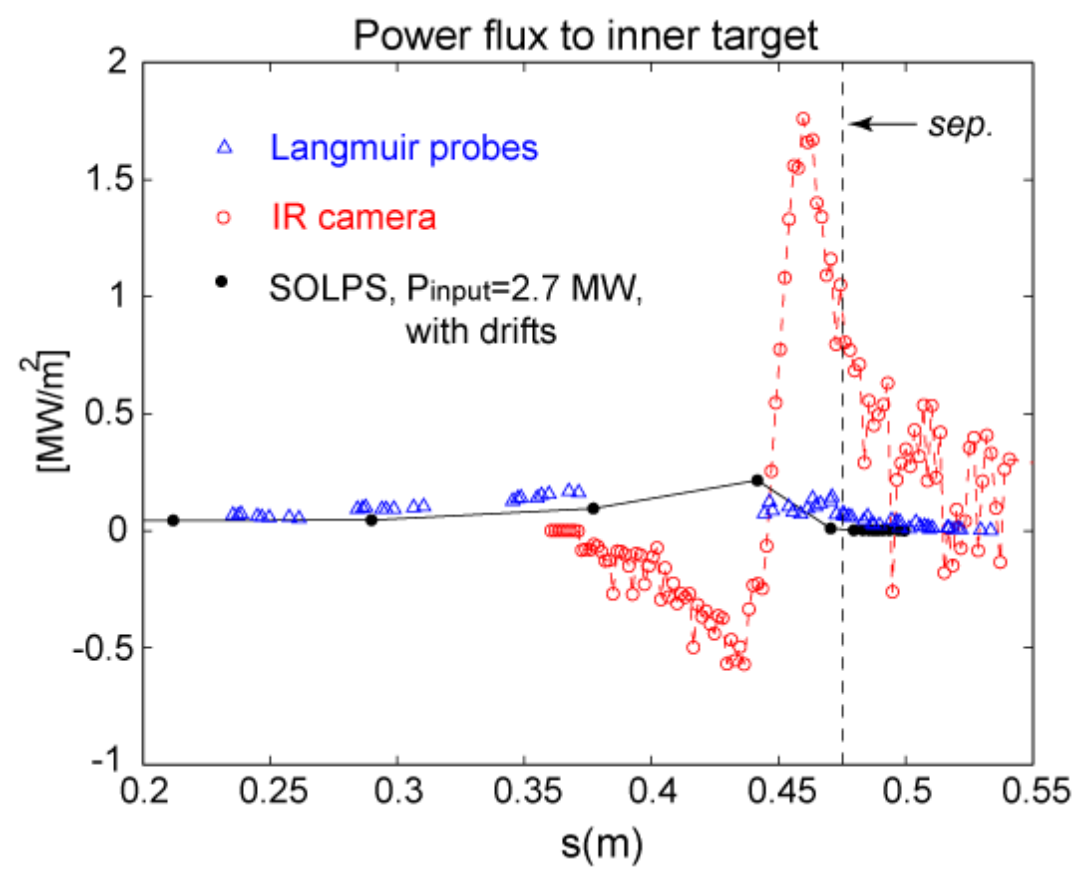

Figure 7. Inner target profiles of power deposition from IR camera, Langmuir probes, and calculated by SOLPS using 2.7 MW input power into the grid with drifts included. Profiles are plotted against the coordinate 's' along the target. 

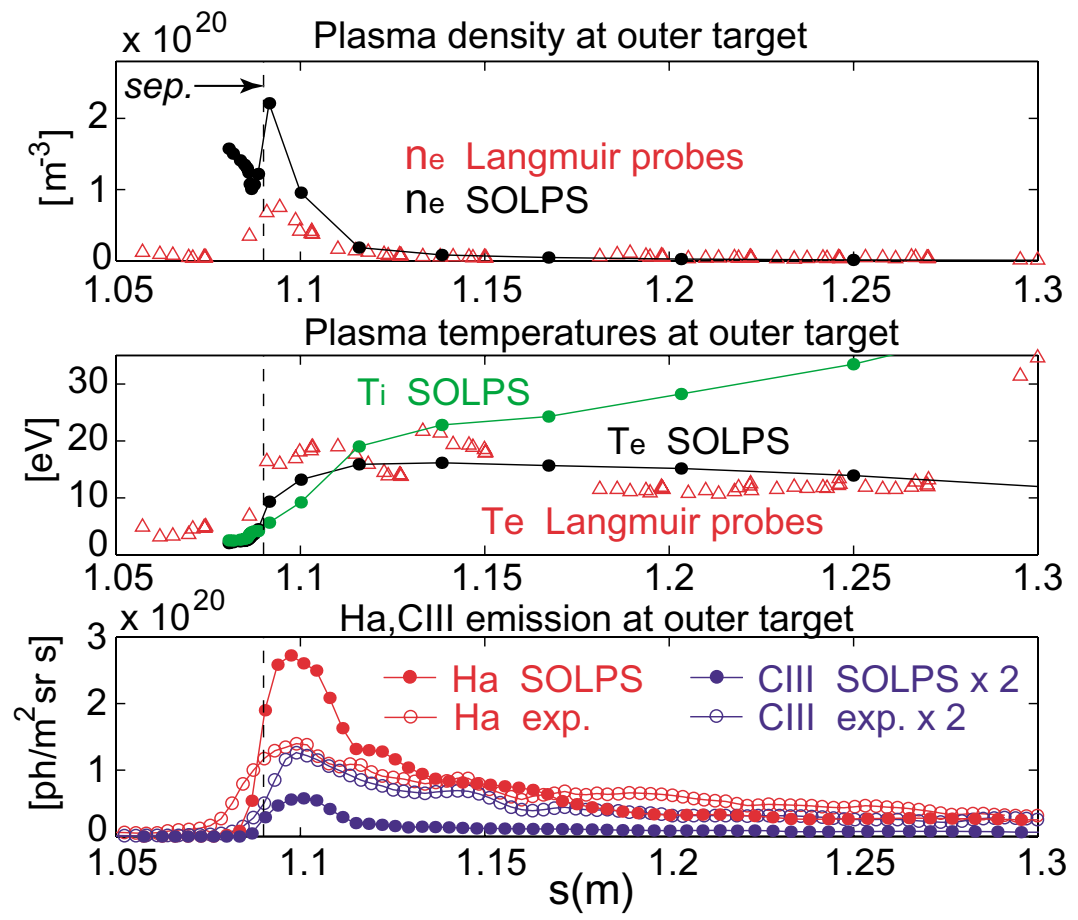

Figure 8. Outer target profiles of measured and calculated electron temperature and density, calculated ion temperature, measured and calculated $H_{\alpha}$ and CIII emissions. The lines of sight for the spectroscopic diagnostics in the divertor are shown in Fig. 9. Profiles are plotted against the coordinate 's' along the target, as in Fig. 5. CIII emissions are scaled by factor 2 for visibility.

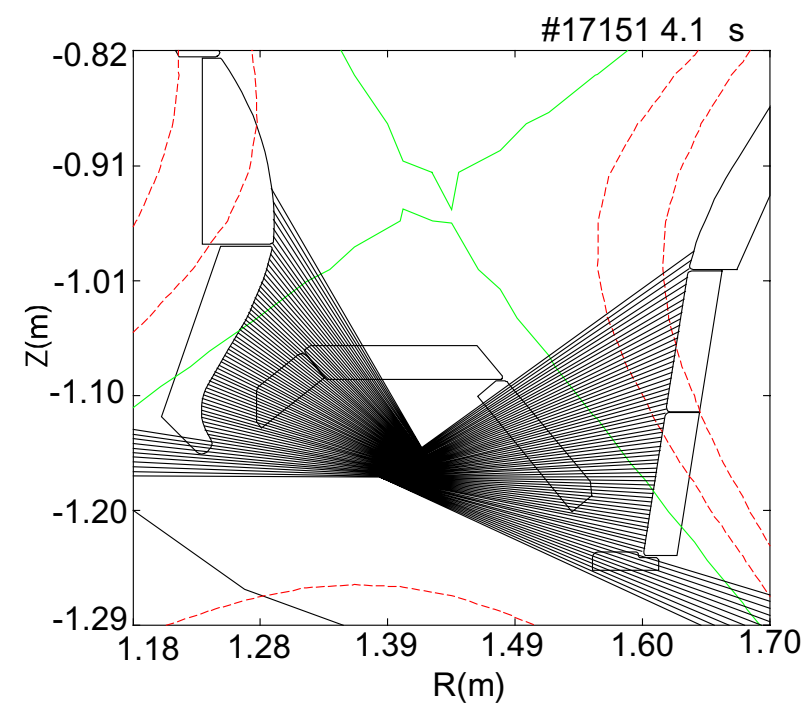

Figure 9. Lines of sight of the divertor spectroscopic diagnostics whose signals, $H_{\alpha}$ and CIII line integrated emissions, are simulated by SOLPS, as shown in Fig. 8. 


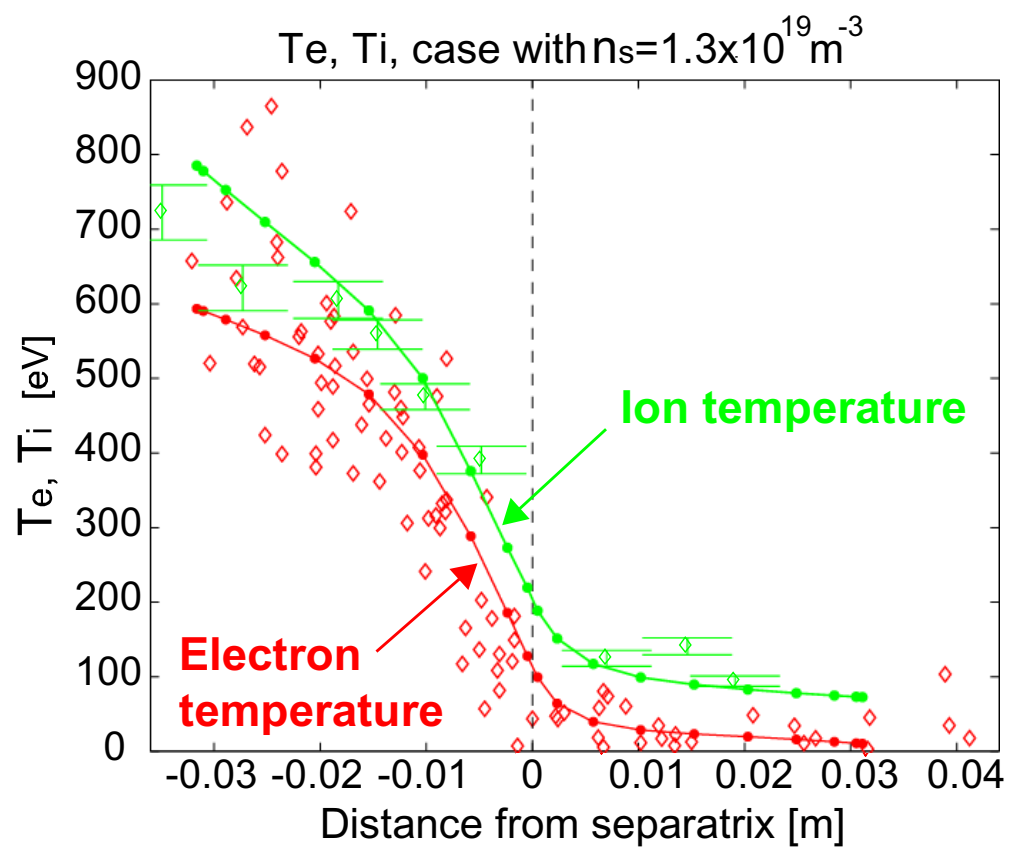

Figure 10. SOLPS fits to experimental $T_{e}$ and $T_{i}$ profiles for the case with reduced separatrix density, $n_{\text {sep }}=1.3 \times 10^{19} \mathrm{~m}^{-3}$.

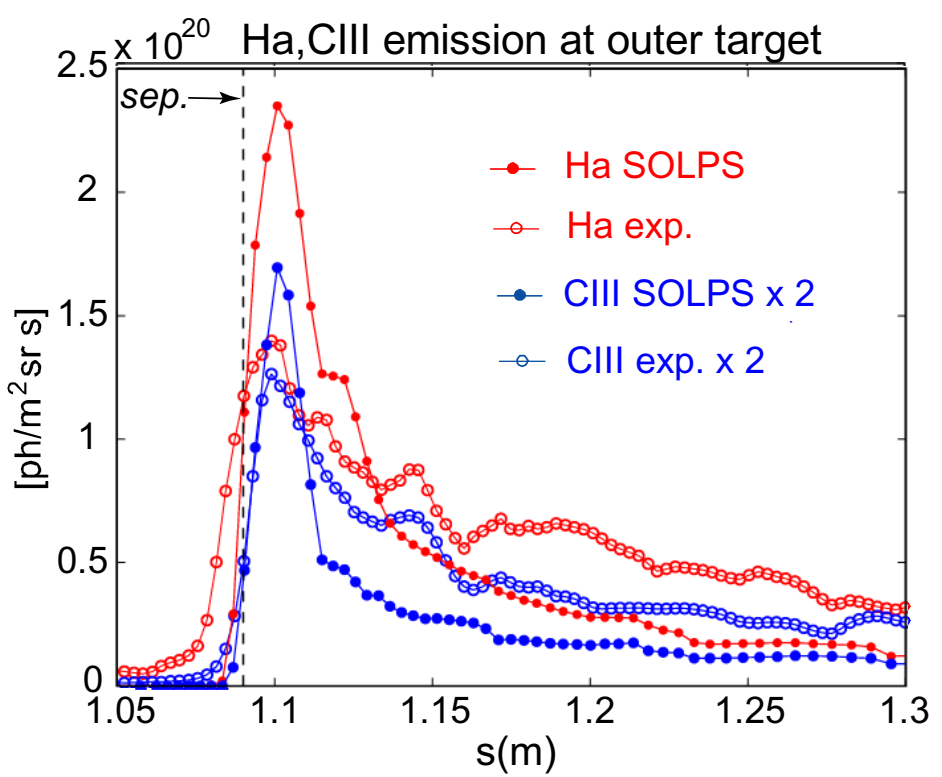

Figure 11. Simulated and measured $H_{\alpha}$ and CIII emission profiles at the outer divertor, for the case with reduced separatrix density, $n_{\text {sep }}=1.3 \times 10^{19} \mathrm{~m}^{-3}$. CIII emissions are scaled by factor 2 for visibility. 

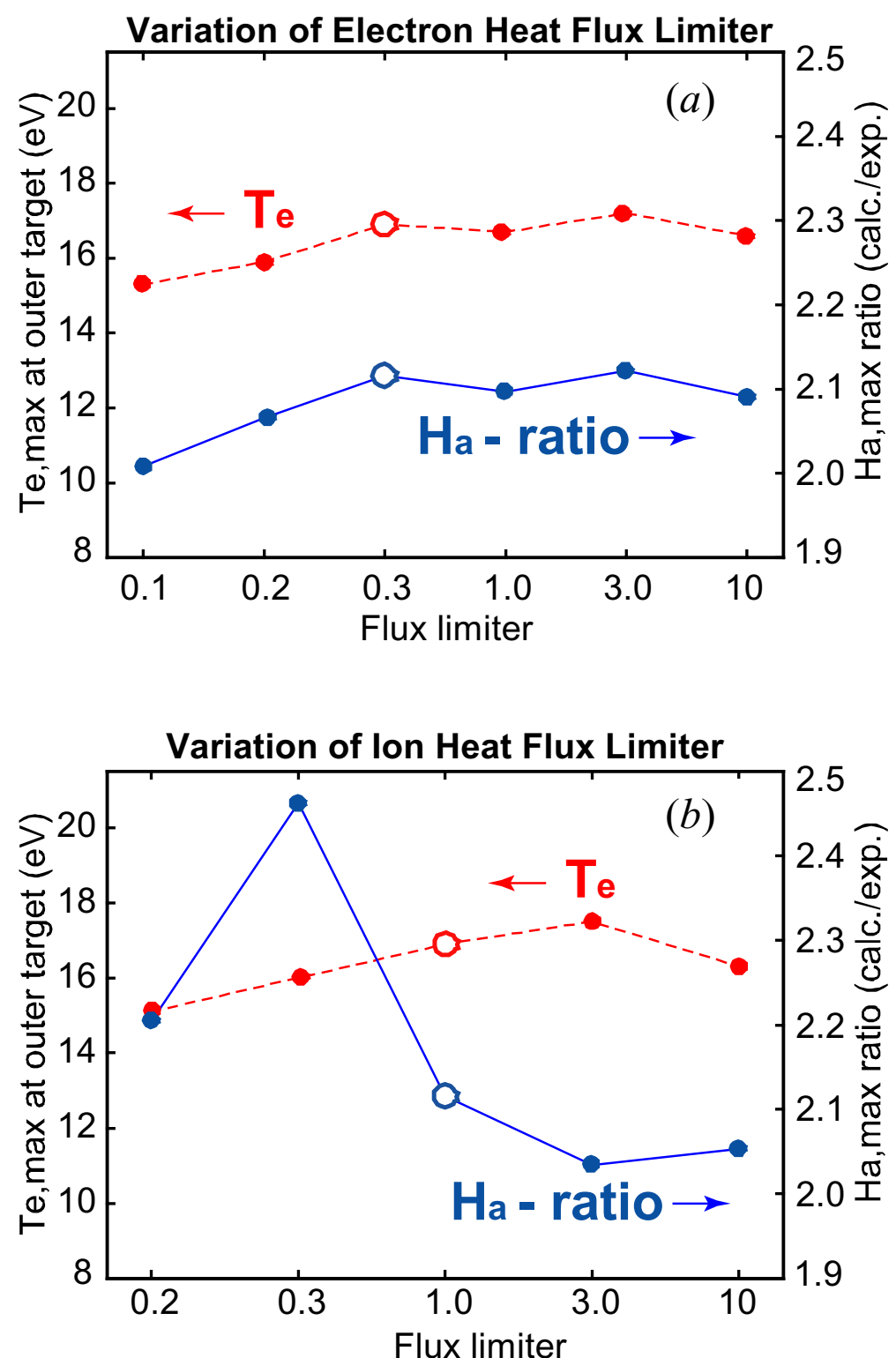

Figure 12. Influence of the variation of electron (a) and ion (b) parallel heat flux limiters on the peak electron temperature at the outer target and the ratio of the peak simulated to experimental $H_{\alpha}$ signals in the outer divertor. The flux limiters are normalized to their values $n_{e} T_{e} v_{\text {th,e }}$ and $n_{i} T_{i} v_{\text {th,i }}$ for electrons and ions, respectively. Open symbols refer to the reference case. 


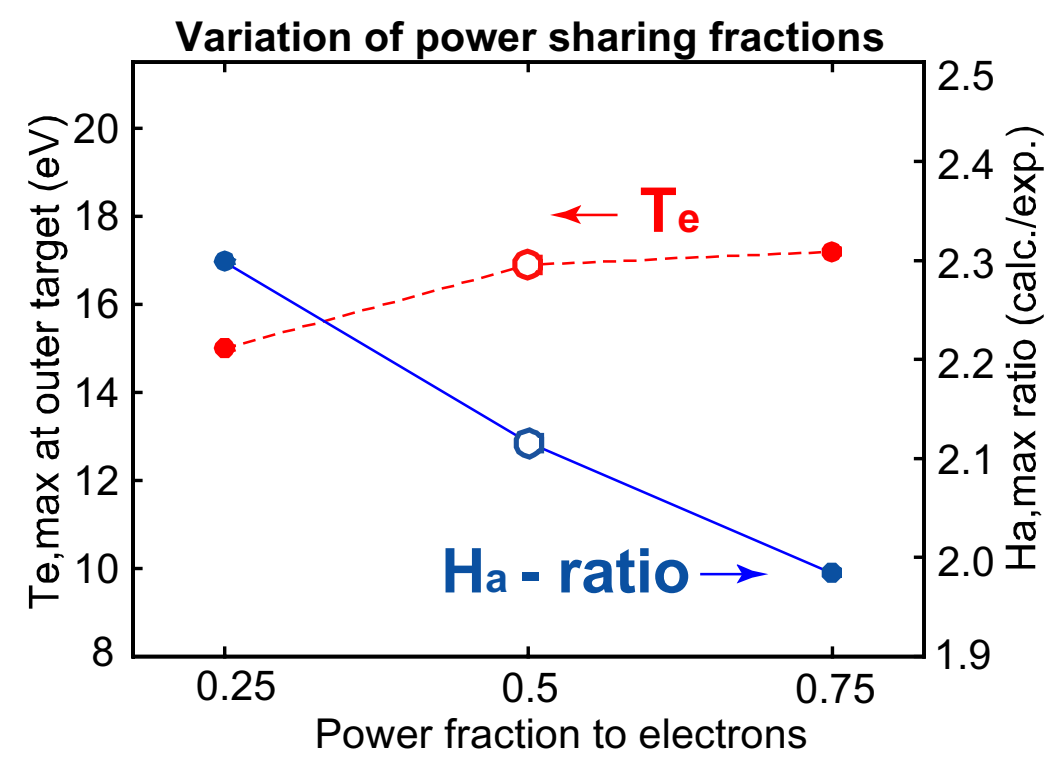

Figure 13. Influence of the variation of input power sharing between ion and electron channels on the peak electron temperature at the outer target and the ratio of peak $H_{\alpha}$ signals (simulated over experimental). Open symbols refer to the reference case.

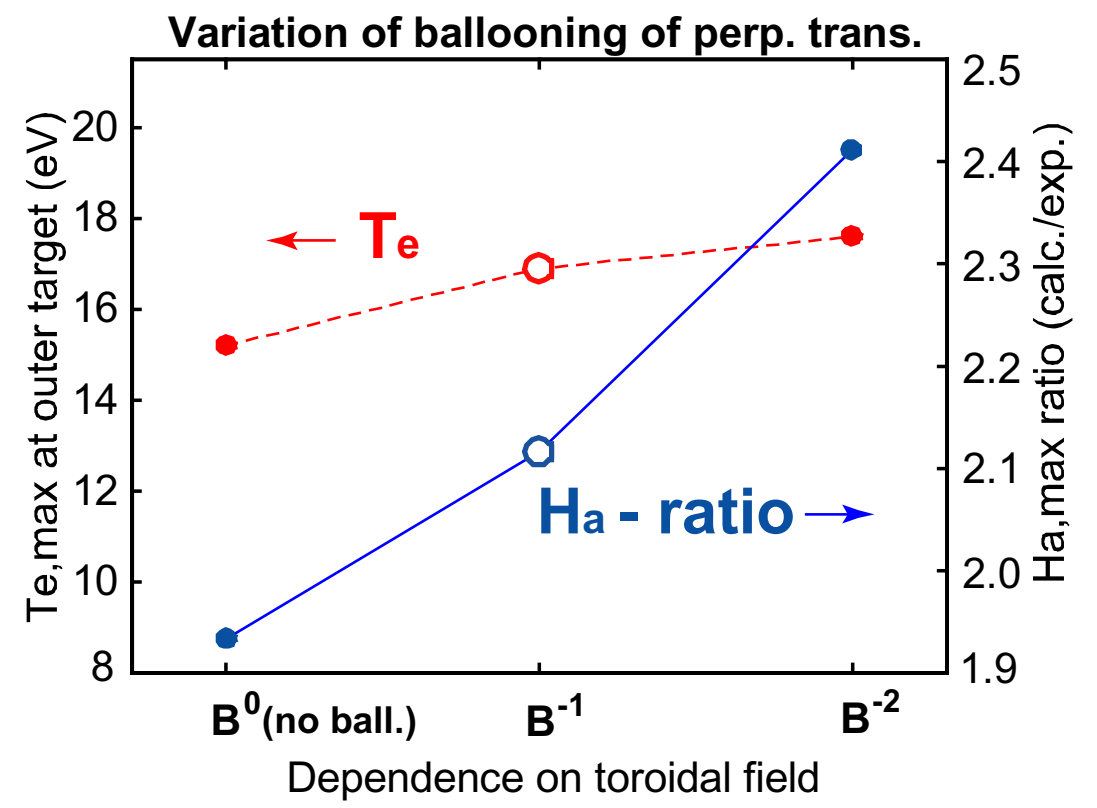

Figure 14. Influence of the variation of degree of 'balloning' of transport coefficients on the peak electron temperature at the outer target and the ratio of peak $H_{\alpha}$ signals (simulated over experimental). Open symbols refer to the reference case. 


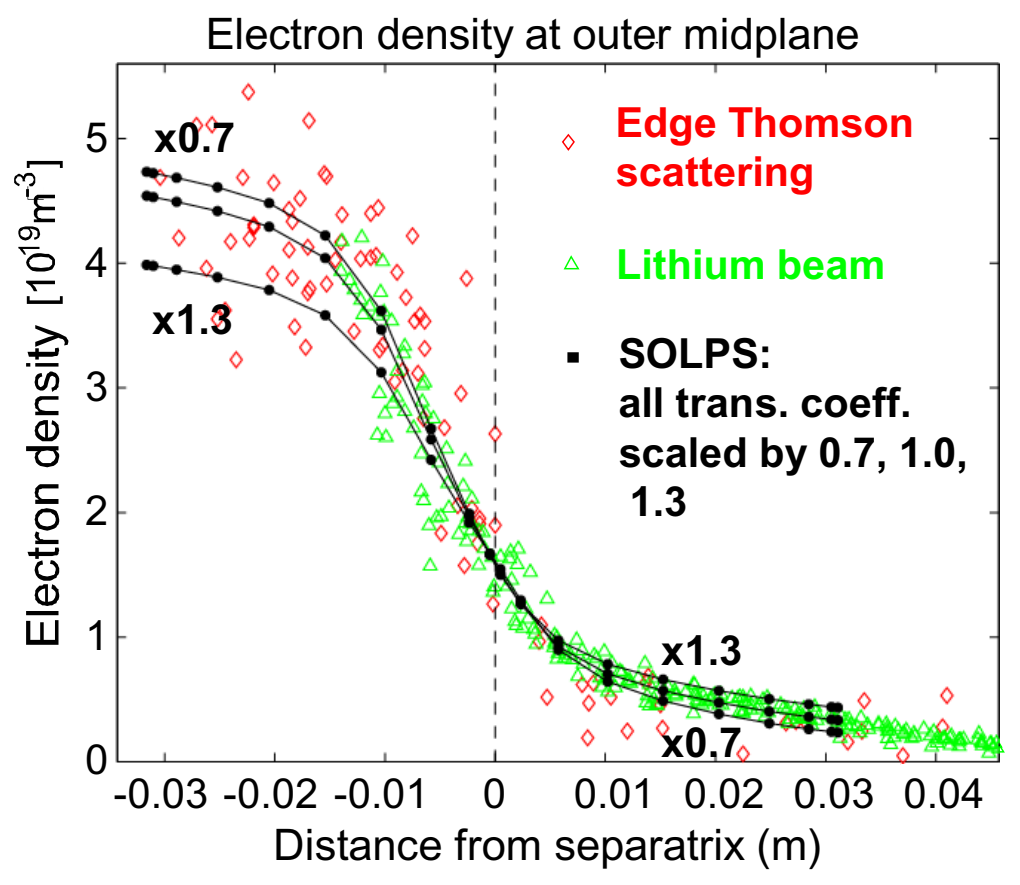

Figure 15. Influence of the variation of transport coefficients in the main SOL on calculated radial density profiles at outer midplane. All coefficients are multiplied by 0.7 and 1.3. Also the SOLPS fit with unchanged transport coefficients is shown for comparison.

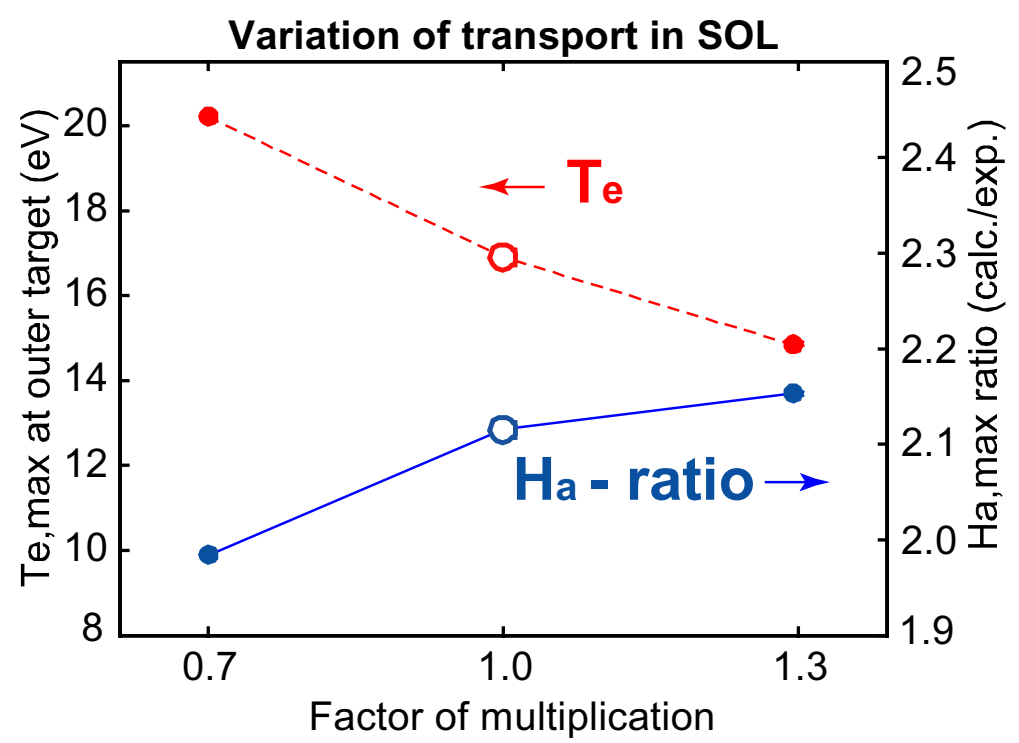

Figure 16. Influence of the variation of transport coefficients in the main SOL on the peak electron temperature at the outer target and the ratio of peak $H_{\alpha}$ signals (simulated over experimental). Open symbols refer to the reference case. 


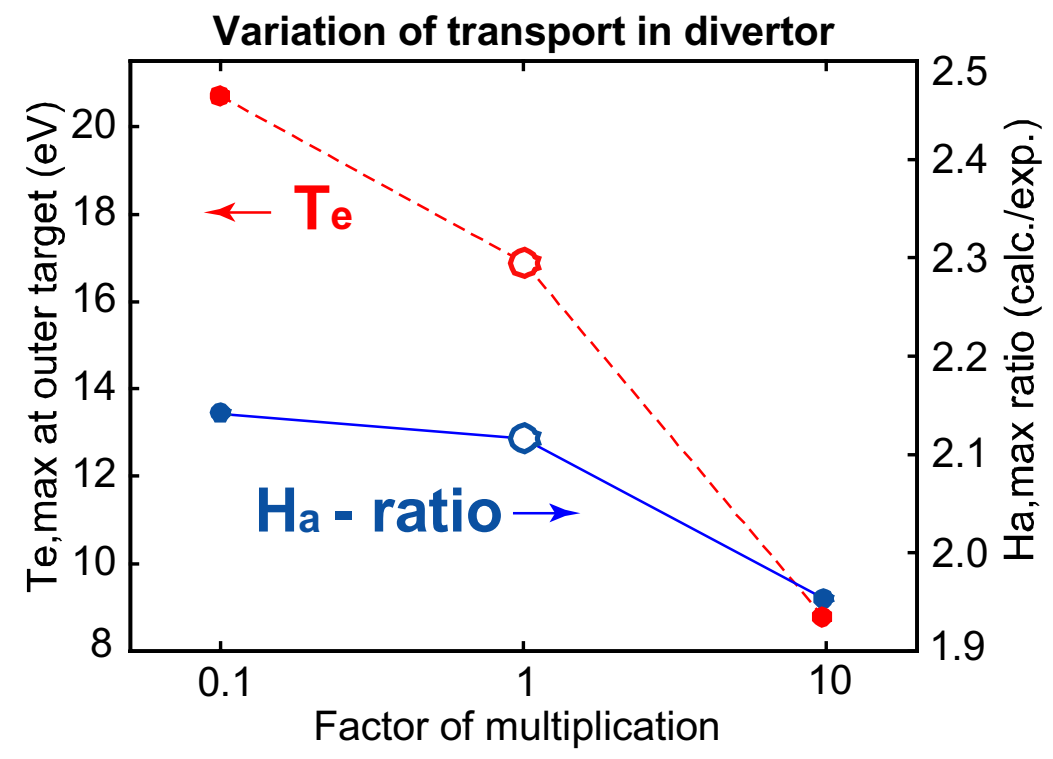

Figure 17. Influence of the variation of transport coefficients in the divertor (common, SOL part) on the peak electron temperature at the outer target and the ratio of peak $H_{\alpha}$ signals (simulated over experimental). Open symbols refer to the reference case.

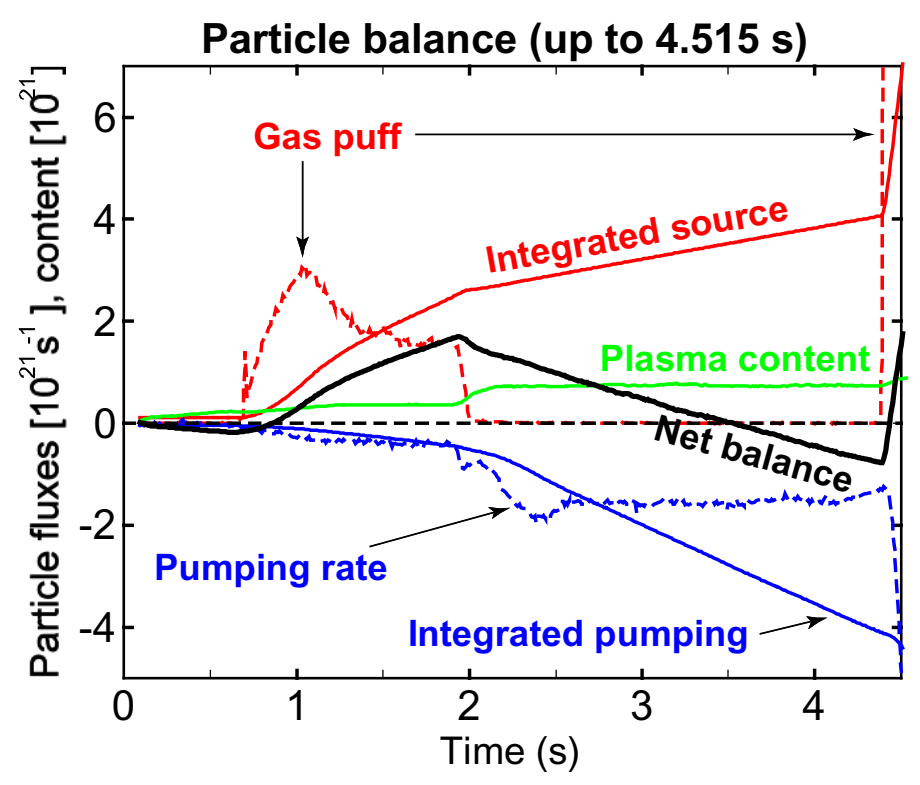

Figure 18. In-vessel particle balance in the shot \#17151, up to the start of a heavy gas puffing. After the initial gas puffing stopped $(a t \approx 2 s)$, the integrated pumping of neutrals exceeded the integrated particle source (mainly from NBI). After $3.6 \mathrm{~s}$, the net particle balance became negative, indicating that the wall has become a net source of particles in the system. 

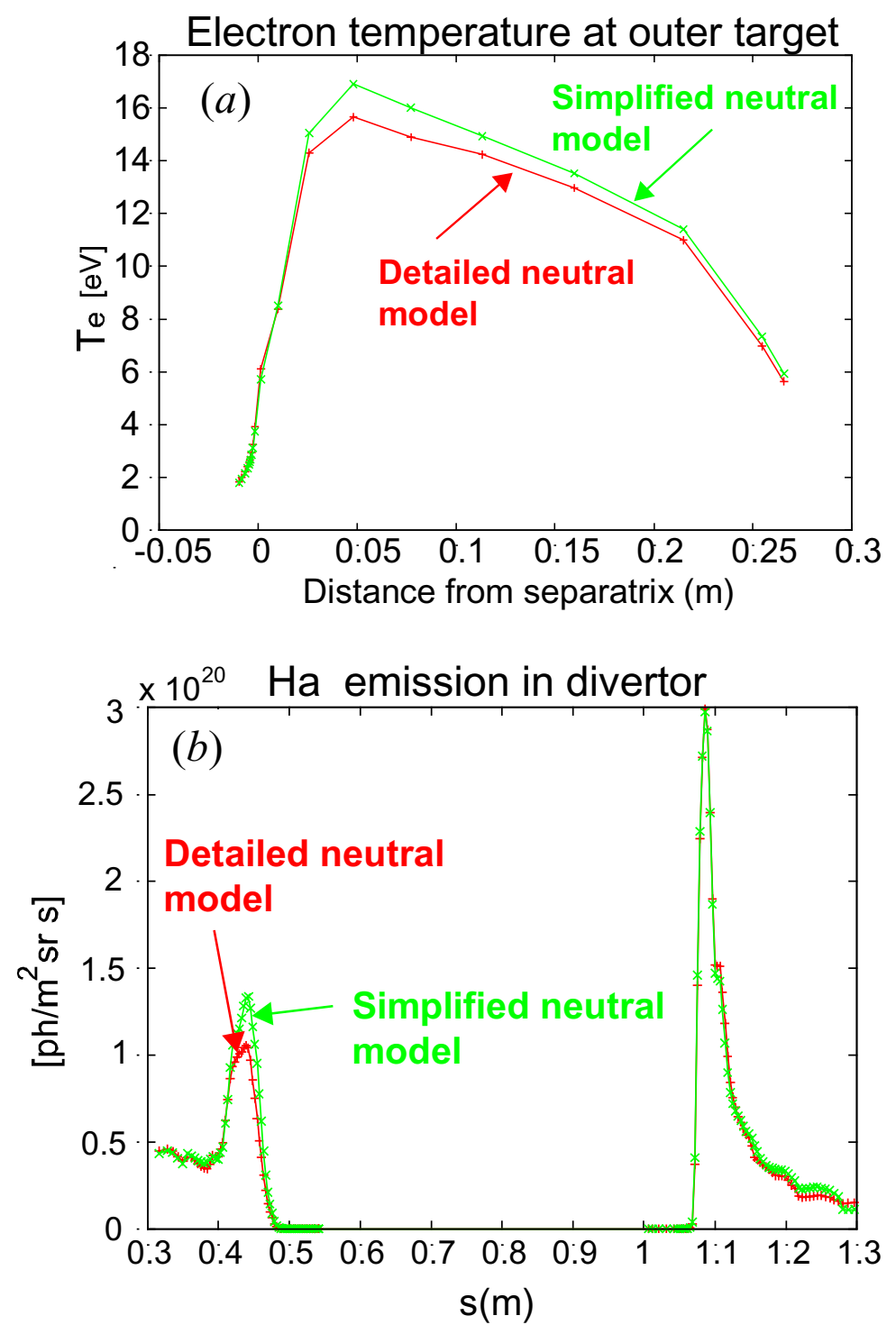

Figure 19. Comparison between outer target electron temperature profiles (a) and $H_{\alpha}$ emission in both inner and outer divertors (b) for the SOLPS cases with simplified and detailed neutral models. 


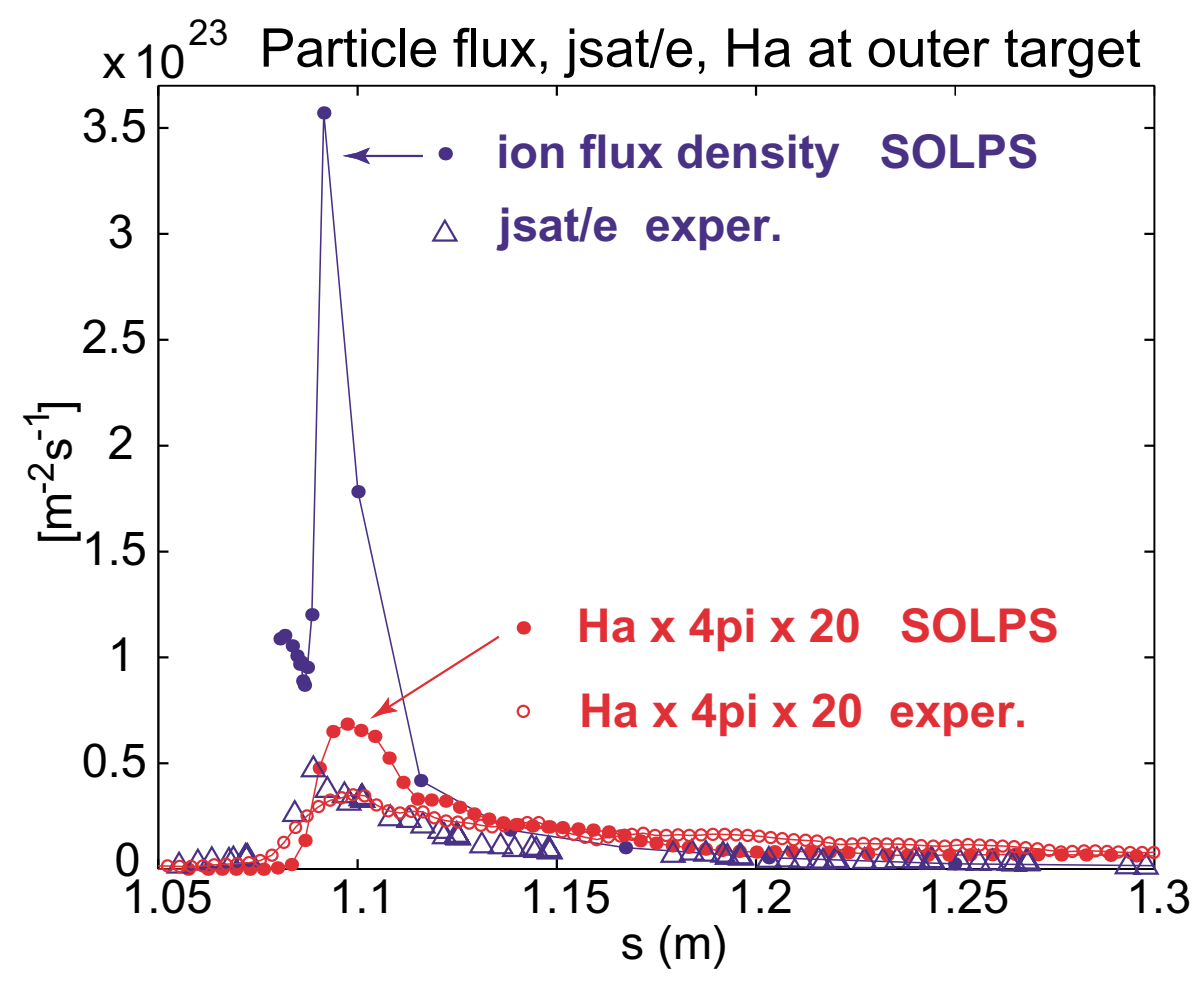

Figure 20. SOLPS simulated and experimental (evaluated from the Langmuir probe ion saturation currents) ion flux densities at the outer target; SOLPS simulated and experimental total $H_{\alpha}$ emissions per unit area multiplied by factor 20. 